\title{
Thermodynamics of continuous media with electromagnetic fields
}

\author{
S.D. Brechet ${ }^{\mathrm{a}}$, F.A. Reuse ${ }^{\mathrm{b}}$, and J.-P. Ansermet ${ }^{\mathrm{c}}$ \\ Institute of Condensed Matter Physics, Station 3, École Polytechnique Fédérale de Lausanne - EPFL, 1015 Lausanne, \\ Switzerland
}

Received 3 August 2012 / Received in final form 24 October 2012

Published online 20 December 2012 - (C) EDP Sciences, Società Italiana di Fisica, Springer-Verlag 2012

\begin{abstract}
The thermodynamics of an electrically charged, multicomponent continuous medium with electromagnetic fields is analysed in the non-relativistic limit. Applying locally the first and second law of thermodynamics and Maxwell's equations for a linear theory of electromagnetism, three equations characterising the continuous medium are derived: a thermostatic equilibrium equation, a reversible and an irreversible thermodynamic evolution equation. For a local thermodynamic equilibrium, explicit expressions for the temperature and the chemical potentials in terms of the electromagnetic fields are obtained. The linear phenomenological relations describe novel effects of non-uniform electromagnetic fields on the transport equations and account also for magnetoresistance and optical tweezers.
\end{abstract}

\section{Introduction}

The development of modern thermodynamics started during the industrialisation period and was closely related to the need to improve the efficiency of steam engines. Since the laws of thermodynamics were discovered in the 19th century, it is commonly believed nowadays that thermodynamics became a mature research field before the dawn of the 20th century. However, this is historically not the case. Since the foundational work of Carnot [1] until after the rise of quantum mechanics, thermodynamics has been a phenomenological theory mainly restricted to the description of either equilibrium states or transformations relating one equilibrium state to another [2]. In essence, thermodynamics was essentially reduced to thermostatics or quasi-thermostatics.

After some pioneering work carried out by Eckart [3,4] in 1940, Stückelberg and Scheurer [5] reformulated thermodynamics as a phenomenological theory describing the evolution of a thermodynamic system by a set of firstorder differential equations in the 1950's. By doing so, he and others following similar lines of thought actually extended the existing theory of equilibrium states or thermostatics, in order to obtain a genuine thermodynamic theory, i.e. a theory describing the evolution and the approach to equilibrium of thermodynamic systems. This extension of thermodynamics is based on the time-dependent state functions "energy" and "entropy" obeying the two fundamental laws of thermodynamics. From these two laws, the time evolution equations of the system are derived.

\footnotetext{
${ }^{a}$ e-mail: sylvain.brechet@epfl.ch

b e-mail: francois.reuse@epfl.ch

c e-mail: jean-philippe.ansermet@epfl.ch
}

By adopting a local description, he also established the local time evolution of a continuous thermodynamic system, thus determining the irreversible thermodynamics of a continuous media. The irreversible thermodynamic framework has notably gained wider recognition through the work of Prigogine [6].

In the present work, we follow essentially the original approach presented in reference [5] and extend the formalism to describe locally the thermodynamics of continuous media with electromagnetic fields. The thermodynamics of fluids with and without electromagnetic fields has been discussed notably by Müller [7] and Muschik et al. [8-12]. In most experimental conditions, the drift velocities of the chemical substances with respect to the laboratory rest frame are clearly non-relativistic. Thus, we restrict our thermodynamic analysis to a non-relativistic linear electromagnetic theory, which corresponds to the electric limit defined by Le Bellac and Lévy-Leblond [13]. Moreover, we limit our analysis to continuous media without intrinsic angular momentum, spontaneous electric polarisation, spontaneous magnetisation or magnetoelectric coupling. We establish explicitly the consistency of a non-relativistic linear electromagnetic theory. In order to obtain the thermostatic equilibrium equation and thermodynamic evolution equations in the local rest frame of the matter, we apply locally the first and second laws on the internal energy of the system using the continuity equations of the relevant dynamical fields. Finally, the linear phenomenological relations $[14,15]$, which are a consequence of the second law, give rise to physical laws and effects coupling generalised currents to generalised forces.

Requiring the local thermodynamic system to be at equilibrium, enables us to define a temperature field and a chemical potential field for the global thermodynamic 
system. We derive explicit expressions for the temperature and the chemical potentials in terms of the electromagnetic fields, which imply that the linear phenomenological relations describe the effects of non-uniform electromagnetic fields on the transport equations. The phenomenological relations account in particular for magnetoresistance and optical tweezers.

The structure of this publication is as follows. In Section 2, we determine the continuity equations of the relevant physical fields based on the axiomatic formulation of the first and second law of thermodynamics outlined in Appendices A.1 and A.2, and the mathematical structure of the continuity equations established in Appendix A.3. In Section 3, we apply these laws to the internal energy of the system and determine the thermostatic and thermodynamic equations. Finally, in Section 4, we characterise physically the linear phenomenological relations.

\section{Thermodynamic continuity equations}

\subsection{Thermodynamic state fields}

The state of a classical continuous medium is defined by a set of state variables. The system is assumed to be continuous on the scale of interest, as explained in Appendix A.3. The local state of a continuous system is defined by a set of state fields that are function of the space and time coordinates. These coordinates are respectively $\left(\mathrm{x}^{\prime}, t^{\prime}\right)$ in the spatial frame, which is the inertial Eulerian frame, and $(\mathbf{x}, t)$ in the local material frame where the center of mass of the local element of matter is at rest, which is the moving Lagrangian frame. The correspondence between the two frames is given in Appendix A.4. In order to determine the local thermodynamic state of a non-relativistic continuous medium consisting of $N$ chemical substances in the presence of electromagnetic fields, we define five types of state fields:

- entropy density $s(\mathbf{x}, t)$,

- densities $n_{A}(\mathbf{x}, t)$ of $N$ chemical substances where $A \in$ $\{1, \ldots, N\}$,

- velocity $\mathbf{v}\left(\mathbf{x}^{\prime}, t\right)$

- electric displacement $\mathbf{D}(\mathbf{x}, t)$,

- magnetic induction $\mathbf{B}(\mathbf{x}, t)$.

Note that the state fields are expressed in terms of the space and time coordinates $(\mathbf{x}, t)$ of the local material frame, except the velocity field $\mathbf{v}\left(\mathbf{x}^{\prime}, t\right)$ that depends on the spatial coordinate $\mathbf{x}^{\prime}$ of the spatial frame, since it represents the relative velocity between the local material frame and the spatial frame, as shown in Appendix A.3.

The local thermodynamic state of the continuous medium is defined formally as the following set of state fields: $\left\{s(\mathbf{x}, t), n_{A}(\mathbf{x}, t), \mathbf{v}\left(\mathbf{x}^{\prime}, t\right), \mathbf{D}(\mathbf{x}, t), \mathbf{B}(\mathbf{x}, t)\right\}$.

The physical fields describing the thermodynamics of such a medium are locally functions of these state fields. Some extensive density fields are of particular interest in this analysis, i.e.

- mass density $m\left(s, n_{A}, \mathbf{v}, \mathbf{D}, \mathbf{B}\right)$,

- electric charge density $q\left(s, n_{A}, \mathbf{v}, \mathbf{D}, \mathbf{B}\right)$,
- energy density $e\left(s, n_{A}, \mathbf{v}, \mathbf{D}, \mathbf{B}\right)$,

- internal energy density $u\left(s, n_{A}, \mathbf{v}, \mathbf{D}, \mathbf{B}\right)$,

- momentum density $\mathbf{p}\left(s, n_{A}, \mathbf{v}, \mathbf{D}, \mathbf{B}\right)$,

- angular momentum density $\ell\left(s, n_{A}, \mathbf{v}, \mathbf{D}, \mathbf{B}\right)$,

where we removed the explicit space and time dependence of the state fields in order to simplify the notation. In the remainder of this publication, we will do likewise.

\subsection{Electromagnetic linear constitutive equations}

We consider a continuous medium without spontaneous electric polarisation or spontaneous magnetisation. In order to have a linear electromagnetic theory, we have to assume the existence of linear mappings between the extensive electromagnetic state fields $\mathbf{D}$ and $\mathbf{B}$ and the intensive electromagnetic fields $\mathbf{E}$ and $\mathbf{H}$, respectively. These mappings are the linear electromagnetic constitutive relations defined in the local material frame of the matter where $\mathbf{v}=\mathbf{0}$. We restrict our analysis to the physical case where $\mathbf{E}$ is independent of $\mathbf{B}$, and $\mathbf{H}$ is independent of $\mathbf{D}$.

In the absence of an electric displacement field $\mathbf{D}$, the electric field $\mathbf{E}\left(s, n_{A}, \mathbf{D}\right)$ vanishes. Similarly, in the absence of a magnetic induction field $\mathbf{B}$, the magnetic field $\mathbf{H}\left(s, n_{A}, \mathbf{B}\right)$ vanishes as well. Thus, the linear electromagnetic constitutive relations in the local material frame are given by,

$$
\begin{aligned}
& \mathbf{E}\left(s, n_{A}, \mathbf{D}\right)=\varepsilon^{-1}\left(s, n_{A}\right) \cdot \mathbf{D}, \\
& \mathbf{H}\left(s, n_{A}, \mathbf{B}\right)=\boldsymbol{\mu}^{-1}\left(s, n_{A}\right) \cdot \mathbf{B},
\end{aligned}
$$

where $\boldsymbol{\varepsilon}\left(s, n_{A}\right)$ and $\boldsymbol{\mu}\left(s, n_{A}\right)$ are, respectively, the electric permittivity and the magnetic permeability tensors of the matter in the local material frame. It is useful to recast the linear electromagnetic constitutive relations (1) explicitly as,

$$
\begin{aligned}
& \mathbf{D}=\boldsymbol{\varepsilon}\left(s, n_{A}\right) \cdot \mathbf{E}\left(s, n_{A}, \mathbf{D}\right), \\
& \mathbf{B}=\boldsymbol{\mu}\left(s, n_{A}\right) \cdot \mathbf{H}\left(s, n_{A}, \mathbf{B}\right) .
\end{aligned}
$$

The electric permittivity tensor $\varepsilon\left(s, n_{A}\right)$ and the magnetic permeability tensor $\boldsymbol{\mu}\left(s, n_{A}\right)$ are recast in terms of the electric susceptibility tensor $\chi_{e}\left(s, n_{A}\right)$ and the magnetic susceptibility tensor $\chi_{m}\left(s, n_{A}\right)$ of the matter, respectively, according to,

$$
\begin{gathered}
\varepsilon\left(s, n_{A}\right)=\varepsilon_{0}\left(\mathbb{1}+\chi_{e}\left(s, n_{A}\right)\right), \\
\boldsymbol{\mu}\left(s, n_{A}\right)=\mu_{0}\left(\mathbb{1}+\chi_{m}\left(s, n_{A}\right)\right),
\end{gathered}
$$

where $\mathbb{1}$ is the rank-2 identity tensor, $\varepsilon_{0}$ and $\mu_{0}$ are, respectively, the electric permittivity and the magnetic permeability in the vacuum that are related by the speed of an electromagnetic wave in the vacuum: $c=1 / \sqrt{\varepsilon_{0} \mu_{0}}$.

\subsection{Thermodynamical continuity equations}

The analytical structure of the continuity equations for extensive physical observables in the local material frame 
is given for scalar fields by the relation (A.22) and for vector fields by the relation (A.23), as established explicitly in Appendix A.3. We now express these equations explicitly in terms of the physical state fields and identify the source and current densities.

\subsubsection{Electric charge continuity equation}

The electric charge density field $q\left(s, n_{A}\right)$ is a property of the matter constituents that is considered as a function of the matter state fields $s$ and $n_{A}$ only. The conservation of the electric charge implies that there is no internal source of charge, i.e. $\rho_{q}=0$. Thus, the continuity equation for the electric charge in the local material frame is given by,

$$
\dot{q}+(\boldsymbol{\nabla} \cdot \mathbf{v}) q+\nabla \cdot \mathbf{j}_{q}=0
$$

where the dot denotes the time derivative in the local material frame, which is related to the time derivative in the spatial frame through the relation (A.20), established in Appendix A.3. Moreover, the diffusive electric current density $\mathbf{j}_{q}$ in the local material frame is related to the electric current density $\mathbf{j}_{q}^{\prime}$ in the spatial frame by,

$$
\mathbf{j}_{q}^{\prime}\left(s, n_{A}, \mathbf{v}\right)=\mathbf{j}_{q}\left(s, n_{A}\right)+q\left(s, n_{A}\right) \mathbf{v},
$$

as shown in Appendix A.4, and the prime denotes a vector field defined in the spatial frame. The physical interpretation of relation (5) is that the electric current density $\mathbf{j}_{q}^{\prime}$ defined in the spatial frame is the sum of the diffusive electric current density $\mathbf{j}_{q}$ defined in the local material frame and the convective electric current density $q \mathbf{v}$ due to the relative motion of the local material frame with respect to the spatial frame.

\subsubsection{Entropy continuity equation}

In order to satisfy locally the evolution condition of the second law (A.2), we require the entropy source density field $\rho_{s}$ to be non-negatively defined, i.e.

$$
\rho_{s} \geqslant 0 \text {. }
$$

The continuity equation for the entropy in the local material frame is given by,

$$
\dot{s}+(\boldsymbol{\nabla} \cdot \mathbf{v}) s+\boldsymbol{\nabla} \cdot \mathbf{j}_{s}=\rho_{s} \geqslant 0 .
$$

\subsubsection{Chemical substance continuity equation}

The source density $\rho_{A}$ of the chemical substance $A$ in a chemical reaction $a$ is proportional to the stoichiometric coefficients $\nu_{a A}$ such that,

$$
\rho_{A}=\sum_{a} \omega_{a} \nu_{a A}
$$

where $\omega_{a}$ is the local rate of the reaction $a$. Note that if the rate of reaction $\omega_{a}$ is positive, then the substances with a positive stoichiometric coefficient $\nu_{a A}$ are produced and the substances with a negative stoichiometric coefficient are consumed, and vice versa if the reaction rate is negative. Thus, the continuity equation for the chemical substance $A$ in the local material frame is given by,

$$
\dot{n}_{A}+(\boldsymbol{\nabla} \cdot \mathbf{v}) n_{A}+\nabla \cdot \mathbf{j}_{A}=\sum_{a} \omega_{a} \nu_{a A} .
$$

\subsubsection{Inhomogeneous Maxwell's equations}

From a mathematical standpoint, the electromagnetic evolution equations, often referred to as Maxwell's equations, are invariant under any diffeomorphism of space-time as first pointed out by van Dantzig [16] and communicated by Dirac. This implies that these equations can be expressed in a fully coordinate-free fashion, for example using the language of differential forms as presented by Misner et al. [17] and showed explicitly by Hehl and Obukhov [18]. In a non-relativistic framework, i.e. $v / c \rightarrow 0$, the diffeomorphism of interest is the Galilean transformation and in a relativistic framework, i.e. $v / c \rightarrow 1$, it is the Lorentz transformation.

It is worth justifying that electromagnetic waves are described consistently within a non-relativistic framework. From a physical perspective, in such a framework, the speed of light in the vacuum, $c$, is infinitely larger than the relative velocity $v$ of the two frames, i.e. $v / c \rightarrow 0$. Thus, in the non-relativistic limit, the speed of light in the vacuum $c$ is the same in the spatial and material frames. This implies that these two frames are equivalent for the description of electromagnetic waves and establishes explicitly the consistency of electromagnetic waves in the non-relativistic limit.

The inhomogeneous Maxwell's equations depend explicitly on the electric charge and current densities. These equations are Gauss' law and Maxwell-Ampère's law defined in the spatial frame respectively as,

$$
\begin{aligned}
\boldsymbol{\nabla}^{\prime} \cdot \mathbf{D}^{\prime} & =q^{\prime}, \\
\partial_{t^{\prime}} \mathbf{D}^{\prime}+\mathbf{j}_{q}^{\prime} & =\nabla^{\prime} \times \mathbf{H}^{\prime},
\end{aligned}
$$

where the prime denotes a scalar field, a vector field or an operator defined in the spatial frame.

As shown in Appendix A.4, the electromagnetic fields $\mathbf{D}$ and $\mathbf{H}$ defined in the local material frame are related to the electromagnetic fields $\mathbf{D}^{\prime}, \mathbf{B}^{\prime}$ and $\mathbf{H}^{\prime}$ defined in the spatial frame by [19],

$$
\begin{aligned}
& \mathbf{D}=\mathbf{D}^{\prime}, \\
& \mathbf{H}\left(s, n_{A}, \mathbf{B}\right)=\mathbf{H}^{\prime}\left(s, n_{A}, \mathbf{v}, \mathbf{B}^{\prime}\right)-\mathbf{v} \times \mathbf{D}^{\prime} .
\end{aligned}
$$

Using the fact that the electric charge density is an invariant, i.e. $q^{\prime}=q$, as well the transformation relations (A.19), (5) and (12), Gauss' law (10) and the vectorial identity,

$$
\begin{aligned}
\boldsymbol{\nabla} \times\left(\mathbf{v} \times \mathbf{D}^{\prime}\right)= & \boldsymbol{\nabla} \times(\mathbf{v} \times \mathbf{D}) \\
= & (\boldsymbol{\nabla} \cdot \mathbf{D}) \mathbf{v}-(\boldsymbol{\nabla} \cdot \mathbf{v}) \mathbf{D} \\
& +(\mathbf{D} \cdot \boldsymbol{\nabla}) \mathbf{v}-(\mathbf{v} \cdot \boldsymbol{\nabla}) \mathbf{D}
\end{aligned}
$$


Gauss' law (10) and Maxwell-Ampère's law (11) are recast respectively in the local material frame as,

$$
\begin{aligned}
& \boldsymbol{\nabla} \cdot \mathbf{D}=q, \\
& \dot{\mathbf{D}}+(\boldsymbol{\nabla} \cdot \mathbf{v}) \mathbf{D}-(\mathbf{D} \cdot \boldsymbol{\nabla}) \mathbf{v}+\mathbf{j}_{q}=\boldsymbol{\nabla} \times \mathbf{H},
\end{aligned}
$$

where Maxwell-Ampère's law (14) represents the continuity equation for the electric displacement field $\mathbf{D}$, which is only valid in the non-relativistic limit, i.e. $v / c \rightarrow 0$. Gauss' law (13) and Maxwell-Ampère's law (14) are Galilean invariant, which can be shown explicitly using the transformation laws established in Appendix A.4.

Note that the continuity equation for the electric charge (4) can be obtained by taking the divergence of Maxwell-Ampère's law (14) and using some vectorial identities.

\subsubsection{Homogeneous Maxwell's equations}

The homogeneous Maxwell's equations do not depend explicitly on the electric charge and current densities. These equations are Thomson's law and Faraday's law defined in the spatial frame, respectively as,

$$
\begin{aligned}
\nabla^{\prime} \cdot \mathbf{B}^{\prime} & =0 \\
\partial_{t^{\prime}} \mathbf{B}^{\prime} & =-\nabla^{\prime} \times \mathbf{E}^{\prime},
\end{aligned}
$$

where the prime denotes a vector field or an operator defined in the spatial frame.

As shown in Appendix A.4, the electromagnetic fields $\mathbf{B}$ and $\mathbf{E}$ defined in the local material frame are related to the electromagnetic fields $\mathbf{D}^{\prime}, \mathbf{B}^{\prime}$ and $\mathbf{E}^{\prime}$ defined in the spatial frame, by [19],

$$
\begin{aligned}
& \mathbf{B}=\mathbf{B}^{\prime}, \\
& \mathbf{E}\left(s, n_{A}, \mathbf{D}\right)=\mathbf{E}^{\prime}\left(s, n_{A}, \mathbf{v}, \mathbf{D}^{\prime}\right)+\mathbf{v} \times \mathbf{B}^{\prime} .
\end{aligned}
$$

Using the transformation relations (A.19) and (17), Thomson's law (15) and the vectorial identity,

$$
\begin{aligned}
\boldsymbol{\nabla} \times\left(\mathbf{v} \times \mathbf{B}^{\prime}\right)= & \boldsymbol{\nabla} \times(\mathbf{v} \times \mathbf{B}) \\
= & (\boldsymbol{\nabla} \cdot \mathbf{B}) \mathbf{v}-(\boldsymbol{\nabla} \cdot \mathbf{v}) \mathbf{B} \\
& +(\mathbf{B} \cdot \boldsymbol{\nabla}) \mathbf{v}-(\mathbf{v} \cdot \boldsymbol{\nabla}) \mathbf{B},
\end{aligned}
$$

Thomson's law (15) and Faraday's law (16) are recast respectively in the local material frame as,

$$
\begin{aligned}
& \boldsymbol{\nabla} \cdot \mathbf{B}=0, \\
& \dot{\mathbf{B}}+(\boldsymbol{\nabla} \cdot \mathbf{v}) \mathbf{B}-(\mathbf{B} \cdot \boldsymbol{\nabla}) \mathbf{v}=-\boldsymbol{\nabla} \times \mathbf{E},
\end{aligned}
$$

where Faraday's law (19) represents the continuity equation for the magnetic induction field $\mathbf{B}$, which is only valid in the non-relativistic limit, i.e. $v / c \rightarrow 0$. Thomson's law (18) and Faraday's law (19) are Galilean invariant, which can be shown explicitly using the transformation laws established in Appendix A.4.

\subsubsection{Energy continuity equation}

In order to satisfy locally the first law (A.1), the only energy source, which does not result from an energy flux, is due to the action of the external forces on the local system. Thus, the energy source density $\rho_{e}$ is of the form,

$$
\rho_{e}=\mathbf{f}^{\text {ext }} \cdot \mathbf{v} \text {. }
$$

In our thermodynamic approach, the electromagnetic force density is exerted by the electromagnetic fields on the matter fields. Since the matter fields and the electromagnetic fields are part of the local system, the electromagnetic force density is considered as an "internal" force of the local system. Thus, the force density $\mathbf{f}^{\text {ext }}$ represents the external force density of the non-electromagnetic fields. The continuity equation for the energy density $e$ is given by,

$$
\dot{e}+(\boldsymbol{\nabla} \cdot \mathbf{v}) e+\boldsymbol{\nabla} \cdot \mathbf{j}_{e}=\mathbf{f}^{\mathrm{ext}} \cdot \mathbf{v} .
$$

The electromagnetic state fields $\mathbf{E}$ and $\mathbf{H}$ are the conjugate fields of the electromagnetic state fields $\mathbf{D}$ and $\mathbf{B}$ respectively, i.e.

$$
\begin{aligned}
& \mathbf{E}\left(s, n_{A}, \mathbf{D}\right)=\frac{\partial e\left(s, n_{A}, \mathbf{v}, \mathbf{D}, \mathbf{B}\right)}{\partial \mathbf{D}}, \\
& \mathbf{H}\left(s, n_{A}, \mathbf{B}\right)=\frac{\partial e\left(s, n_{A}, \mathbf{v}, \mathbf{D}, \mathbf{B}\right)}{\partial \mathbf{B}} .
\end{aligned}
$$

The Schwarz integrability conditions for the energy density,

$$
\begin{aligned}
& \frac{\partial}{\partial D^{i}}\left(\frac{\partial e}{\partial D^{j}}\right)=\frac{\partial}{\partial D^{j}}\left(\frac{\partial e}{\partial D^{i}}\right), \\
& \frac{\partial}{\partial B^{i}}\left(\frac{\partial e}{\partial B^{j}}\right)=\frac{\partial}{\partial B^{j}}\left(\frac{\partial e}{\partial B^{i}}\right),
\end{aligned}
$$

the definitions (22) and the linear electromagnetic constitutive equations (2) imply that the electric permittivity tensor $\varepsilon\left(s, n_{A}\right)$ and the magnetic permeability tensor $\boldsymbol{\mu}\left(s, n_{A}\right)$ are symmetric with respect to their indices. Moreover, the definitions (3) imply that the electric susceptibility tensor $\chi_{e}\left(s, n_{A}\right)$ and the magnetic susceptibility tensor $\chi_{m}\left(s, n_{A}\right)$ are also symmetric with respect to their indices.

The analytical expression for the energy density $e\left(s, n_{A}, \mathbf{v}, \mathbf{D}, \mathbf{B}\right)$ can be split as,

$$
e\left(s, n_{A}, \mathbf{v}, \mathbf{D}, \mathbf{B}\right)=e^{\mathrm{mat}}\left(s, n_{A}, \mathbf{v}\right)+e^{\mathrm{em}}\left(s, n_{A}, \mathbf{D}, \mathbf{B}\right),
$$

where $e^{\text {mat }}\left(s, n_{A}, \mathbf{v}\right) \equiv e\left(s, n_{A}, \mathbf{v}, \mathbf{0}, \mathbf{0}\right)$ is the energy density of the matter fields and $e^{\mathrm{em}}\left(s, n_{A}, \mathbf{D}, \mathbf{B}\right) \equiv$ $e\left(s, n_{A}, \mathbf{0}, \mathbf{D}, \mathbf{B}\right)$ is the energy density of the electromagnetic fields and of their interaction with the matter fields, which has to satisfy the integrability conditions (22), i.e.

$$
\begin{aligned}
& e^{\mathrm{em}}\left(s, n_{A}, \mathbf{D}, \mathbf{B}\right)= \\
& \frac{1}{2}\left(\varepsilon^{-1}\left(s, n_{A}\right) \cdot(\mathbf{D} \odot \mathbf{D})+\boldsymbol{\mu}^{-1}\left(s, n_{A}\right) \cdot(\mathbf{B} \odot \mathbf{B})\right),
\end{aligned}
$$


where the symbol $\odot$ denotes a symmetrised tensorial product $\otimes$. Thus, the electromagnetic energy density is a positive definite quadratic form, as expected for a linear electromagnetic theory. It can be written explicitly in components as,

$$
\begin{aligned}
& e^{\mathrm{em}}\left(s, n_{A}, \mathbf{D}, \mathbf{B}\right)= \\
& \frac{1}{2}\left(\varepsilon_{i j}^{-1}\left(s, n_{A}\right) D^{i} D^{j}+\mu_{i j}^{-1}\left(s, n_{A}\right) B^{i} B^{j}\right),
\end{aligned}
$$

using the Einstein implicit summation convention.

Using the linear electromagnetic constitutive equation (2), it is convenient to recast the electromagnetic energy density (25) as,

$$
e^{\mathrm{em}}\left(s, n_{A}, \mathbf{D}, \mathbf{B}\right)=\frac{1}{2}(\mathbf{E} \cdot \mathbf{D}+\mathbf{H} \cdot \mathbf{B}) .
$$

The energy density $e\left(s, n_{A}, \mathbf{v}, \mathbf{D}, \mathbf{B}\right)$ can be split into a kinetic and an internal part according to,

$$
e\left(s, n_{A}, \mathbf{v}, \mathbf{D}, \mathbf{B}\right)=\frac{1}{2} m\left(s, n_{A}\right) \mathbf{v}^{2}+u\left(s, n_{A}, \mathbf{D}, \mathbf{B}\right),
$$

where $u\left(s, n_{A}, \mathbf{D}, \mathbf{B}\right)=e\left(s, n_{A}, \mathbf{0}, \mathbf{D}, \mathbf{B}\right)$ is the internal energy density, which is defined as the energy density in the local material frame where $\mathbf{v}=\mathbf{0}$.

The internal energy density $u\left(s, n_{A}, \mathbf{D}, \mathbf{B}\right)$ can be split as,

$$
u\left(s, n_{A}, \mathbf{D}, \mathbf{B}\right)=u^{\mathrm{mat}}\left(s, n_{A}\right)+u^{\mathrm{em}}\left(s, n_{A}, \mathbf{D}, \mathbf{B}\right),
$$

where $u^{\text {mat }}\left(s, n_{A}\right) \equiv u\left(s, n_{A}, \mathbf{0}, \mathbf{0}\right)$ is the internal energy density of the matter fields and $u^{\mathrm{em}}\left(s, n_{A}, \mathbf{D}, \mathbf{B}\right)$ is the internal energy density of the electromagnetic fields and of their interaction with the matter fields, which is given by,

$$
\begin{aligned}
u^{\mathrm{em}}\left(s, n_{A}, \mathbf{D}, \mathbf{B}\right)= & \frac{1}{2}\left(\varepsilon^{-1}\left(s, n_{A}\right) \cdot(\mathbf{D} \odot \mathbf{D})\right. \\
& \left.+\boldsymbol{\mu}^{-1}\left(s, n_{A}\right) \cdot(\mathbf{B} \odot \mathbf{B})\right) .
\end{aligned}
$$

Since $u^{\mathrm{em}}\left(s, n_{A}, \mathbf{D}, \mathbf{B}\right)$ is a quadratic form of the frameindependent vectors fields $\mathbf{D}$ and $\mathbf{B}$, it is invariant under translations, as expected.

Using the constitutive electromagnetic relation (2), it is convenient to recast the electromagnetic part of the internal energy density (29) as,

$$
u^{\mathrm{em}}\left(s, n_{A}, \mathbf{D}, \mathbf{B}\right)=\frac{1}{2}(\mathbf{E} \cdot \mathbf{D}+\mathbf{H} \cdot \mathbf{B}) .
$$

Since the electromagnetic fields in the local material frame are independent of the velocity field $\mathbf{v}$, the relation (27) implies that the electromagnetic fields $\mathbf{E}\left(s, n_{A}, \mathbf{D}\right)$ and $\mathbf{H}\left(s, n_{A}, \mathbf{B}\right)$ are the conjugate fields of the electromagnetic state fields $\mathbf{D}$ and $\mathbf{B}$ with respect to the internal energy density $u\left(s, n_{A}, \mathbf{D}, \mathbf{B}\right)$, i.e.

$$
\begin{aligned}
\mathbf{E}\left(s, n_{A}, \mathbf{D}\right) & =\frac{\partial u\left(s, n_{A}, \mathbf{D}, \mathbf{B}\right)}{\partial \mathbf{D}}, \\
\mathbf{H}\left(s, n_{A}, \mathbf{B}\right) & =\frac{\partial u\left(s, n_{A}, \mathbf{D}, \mathbf{B}\right)}{\partial \mathbf{B}} .
\end{aligned}
$$

\subsubsection{Momentum and mass continuity equations}

The momentum source density is due to the external force density only, i.e.

$$
\rho_{\mathbf{p}}=\mathbf{f}^{\mathrm{ext}},
$$

where $\mathbf{f}^{\text {ext }}$ is the external force density. The continuity equation for the momentum density $\mathbf{p}$ is given by,

$$
\dot{\mathbf{p}}+(\boldsymbol{\nabla} \cdot \mathbf{v}) \mathbf{p}-\nabla \cdot \boldsymbol{\sigma}=\mathbf{f}^{\mathrm{ext}}
$$

where the stress tensor $\boldsymbol{\sigma}$ is defined as the opposite of the momentum current density tensor $j_{\mathbf{p}}$, i.e.

$$
\boldsymbol{\sigma} \equiv-j_{\mathbf{p}}
$$

The momentum density is of the form [20],

$$
\mathbf{p}\left(s, n_{A}, \mathbf{v}, \mathbf{D}, \mathbf{B}\right)=m\left(s, n_{A}\right) \mathbf{v}+\mathbf{D} \times \mathbf{B} .
$$

It is useful to split the momentum density (35) into a matter part $\mathbf{p}^{\text {mat }}\left(s, n_{A}, \mathbf{v}\right)$ and an electromagnetic part $\mathbf{p}^{\mathrm{em}}(\mathbf{D}, \mathbf{B})$ according to,

$$
\mathbf{p}\left(s, n_{A}, \mathbf{v}, \mathbf{D}, \mathbf{B}\right)=\mathbf{p}^{\mathrm{mat}}\left(s, n_{A}, \mathbf{v}\right)+\mathbf{p}^{\mathrm{em}}(\mathbf{D}, \mathbf{B}),
$$

where

$$
\begin{aligned}
& \mathbf{p}^{\text {mat }}\left(s, n_{A}, \mathbf{v}\right)=m\left(s, n_{A}\right) \mathbf{v} \\
& \mathbf{p}^{\mathrm{em}}(\mathbf{D}, \mathbf{B})=\mathbf{D} \times \mathbf{B}
\end{aligned}
$$

Similarly, the stress tensor $\boldsymbol{\sigma}\left(s, n_{A}, \mathbf{v}, \mathbf{D}, \mathbf{B}\right)$ is also split into a matter part $\boldsymbol{\sigma}^{\text {mat }}\left(s, n_{A}, \mathbf{v}\right)$ and an electromagnetic part $\boldsymbol{\sigma}^{\mathrm{em}}\left(s, n_{A}, \mathbf{D}, \mathbf{B}\right)$ according to,

$$
\boldsymbol{\sigma}\left(s, n_{A}, \mathbf{v}, \mathbf{D}, \mathbf{B}\right)=\boldsymbol{\sigma}^{\mathrm{mat}}\left(s, n_{A}, \mathbf{v}\right)+\boldsymbol{\sigma}^{\mathrm{em}}\left(s, n_{A}, \mathbf{D}, \mathbf{B}\right) .
$$

The relations (36) and (39) imply that the continuity equation (33) can be split according to,

$$
\begin{aligned}
& \dot{\mathbf{p}}^{\mathrm{mat}}+(\boldsymbol{\nabla} \cdot \mathbf{v}) \mathbf{p}^{\mathrm{mat}}-\boldsymbol{\nabla} \cdot \boldsymbol{\sigma}^{\mathrm{mat}}=\mathbf{f}^{\mathrm{ext}}+\mathbf{f}^{\mathrm{em}}, \\
& \dot{\mathbf{p}}^{\mathrm{em}}+(\boldsymbol{\nabla} \cdot \mathbf{v}) \mathbf{p}^{\mathrm{em}}-\boldsymbol{\nabla} \cdot \boldsymbol{\sigma}^{\mathrm{em}}=-\mathbf{f}^{\mathrm{em}}
\end{aligned}
$$

where $\mathbf{f}^{\mathrm{em}}$ is the electromagnetic force density exerted by the electromagnetic fields on the matter fields. Note that the electromagnetic force density $\mathbf{f}^{\mathrm{em}}$ is an internal force density for the whole system. However, it is considered as an "external" force density for the matter or electromagnetic subsystems.

Using the expression (37) for the momentum density of the matter part, the continuity equation (40) for the momentum density of the matter part becomes,

$$
m \dot{\mathbf{v}}+(\dot{m}+(\boldsymbol{\nabla} \cdot \mathbf{v}) m) \mathbf{v}=\mathbf{f}^{\text {ext }}+\mathbf{f}^{\mathrm{em}}+\boldsymbol{\nabla} \cdot \boldsymbol{\sigma}^{\mathrm{mat}},
$$

which is Newton's second law of motion. The nonrelativistic frame-independence of Newton's second law of motion implies that the second term on the LHS of the equation of motion (42) has to vanish, i.e.

$$
\dot{m}+(\boldsymbol{\nabla} \cdot \mathbf{v}) m=0,
$$


which is the continuity equation for the mass. Since the mass density field $m\left(s, n_{A}\right)$ is a property of the matter constituents, it is a function of the matter scalar state fields $s$ and $n_{A}$ only. The continuity equation (43) for the mass implies that there is no internal source of mass, i.e. $\rho_{m}=0$, and no diffusive mass current density, i.e. $\mathbf{j}_{m}=$ 0. Physically, this implies that the mass is a conserved quantity in a non-relativistic framework.

Using the continuity equation (43), Newton's second law of motion reduces to,

$$
m \dot{\mathbf{v}}=\mathbf{f}^{\mathrm{ext}}+\mathbf{f}^{\mathrm{em}}+\boldsymbol{\nabla} \cdot \boldsymbol{\sigma}^{\mathrm{mat}},
$$

and corresponds to one of the Navier-Stokes equations, expressed in the local material frame, for an electrically charged continuous medium with electromagnetic fields. Note that the term $\boldsymbol{\nabla} \cdot \boldsymbol{\sigma}^{\text {mat }}$ represents the mechanical shear force density.

The expressions for the electromagnetic constitutive equations (2), the susceptibility tensors (3) and the electromagnetic momentum density (38) imply that the scalar product,

$$
\begin{aligned}
\left(\dot{\mathbf{p}}^{\mathrm{em}}+(\boldsymbol{\nabla} \cdot \mathbf{v}) \mathbf{p}^{\mathrm{em}}\right) \cdot \mathbf{v}= & \frac{\mathbf{v}}{c} \cdot \frac{1}{c}\left[\left(\left(\left(\mathbb{1}+\boldsymbol{\chi}_{e}\right) \cdot \mathbf{E}\right)\right.\right. \\
& \left.\times\left(\left(\mathbb{1}+\boldsymbol{\chi}_{m}\right) \cdot \mathbf{H}\right)\right) \\
& +(\boldsymbol{\nabla} \cdot \mathbf{v})\left(\left(\left(\mathbb{1}+\boldsymbol{\chi}_{e}\right) \cdot \mathbf{E}\right)\right. \\
& \left.\left.\times\left(\left(\mathbb{1}+\boldsymbol{\chi}_{m}\right) \cdot \mathbf{H}\right)\right)\right]
\end{aligned}
$$

is a relativistic correction proportional to $v / c$, which is neglected in a non-relativistic framework and the dot on the righthand corner of the brackets on the second line denotes a material time derivative. Thus, in the non-relativistic limit, the scalar product of the continuity equation (41) and the velocity vector $\mathbf{v}$ reduces to,

$$
\left(\boldsymbol{\nabla} \cdot \boldsymbol{\sigma}^{\mathrm{em}}\right) \cdot \mathbf{v}=\mathbf{f}^{\mathrm{em}} \cdot \mathbf{v} .
$$

Maxwell-Ampère's law (14) and Faraday's law (19) imply that the scalar product of the continuity equation (41) and the velocity field $\mathbf{v}$ can be expressed as,

$$
\begin{aligned}
& \left((\mathbf{D} \times \mathbf{B})^{\cdot}+2(\boldsymbol{\nabla} \cdot \mathbf{v})(\mathbf{D} \times \mathbf{B})\right. \\
& -((\mathbf{D} \cdot \boldsymbol{\nabla}) \mathbf{v}) \times \mathbf{B}-\mathbf{D} \times((\mathbf{B} \cdot \boldsymbol{\nabla}) \mathbf{v})) \cdot \mathbf{v} \\
& =\left(-\mathbf{j}_{q} \times \mathbf{B}-\mathbf{D} \times(\boldsymbol{\nabla} \times \mathbf{E})-\mathbf{B} \times(\boldsymbol{\nabla} \times \mathbf{H})\right) \cdot \mathbf{v}
\end{aligned}
$$

where the terms on the LHS are relativistic corrections that are neglected in a non-relativistic framework and the dot on the righthand corner of the first brackets on the first line denotes a material time derivative. Thus, in the non-relativistic limit, the scalar product (46) reduces to,

$$
(-\mathbf{D} \times(\boldsymbol{\nabla} \times \mathbf{E})-\mathbf{B} \times(\boldsymbol{\nabla} \times \mathbf{H})) \cdot \mathbf{v}=\left(\mathbf{j}_{q} \times \mathbf{B}\right) \cdot \mathbf{v} .
$$

Using the vectorial identities,

$$
\begin{aligned}
& -\mathbf{D} \times(\boldsymbol{\nabla} \times \mathbf{E})=-\mathbf{D} \nabla \mathbf{E}+(\mathbf{D} \cdot \boldsymbol{\nabla}) \mathbf{E} \\
& =-\mathbf{D} \nabla \mathbf{E}-(\boldsymbol{\nabla} \cdot \mathbf{D}) \mathbf{E}+\nabla_{\mathbf{D}} \cdot(\mathbf{D} \odot \mathbf{E}),
\end{aligned}
$$

and

$$
\begin{aligned}
& -\mathbf{B} \times(\boldsymbol{\nabla} \times \mathbf{H})=-\mathbf{B} \nabla \mathbf{H}+(\mathbf{B} \cdot \boldsymbol{\nabla}) \mathbf{H} \\
& =-\mathbf{B} \nabla \mathbf{H}-(\boldsymbol{\nabla} \cdot \mathbf{B}) \mathbf{H}+\nabla_{\mathbf{B}} \cdot(\mathbf{B} \odot \mathbf{H}),
\end{aligned}
$$

and Gauss' law (13) and Thomson's law (18), the equation (47) is recast as,

$$
\begin{aligned}
& \left(\nabla_{\mathbf{D}, \mathbf{B}} \cdot(\mathbf{D} \odot \mathbf{E}+\mathbf{B} \odot \mathbf{H})\right) \cdot \mathbf{v} \\
& =\left(q \mathbf{E}+\mathbf{j}_{q} \times \mathbf{B}+\mathbf{D} \nabla \mathbf{E}+\mathbf{B} \nabla \mathbf{H}\right) \cdot \mathbf{v},
\end{aligned}
$$

where the covariant vectors $\boldsymbol{\nabla}_{\mathbf{D}, \mathbf{B}} \cdot(\mathbf{D} \odot \mathbf{E}+\mathbf{B} \odot \mathbf{H})$ and $\mathbf{D} \nabla \mathbf{E}+\mathbf{B} \nabla \mathbf{H}$ are explicitly written in components as,

$$
\begin{aligned}
& \boldsymbol{\nabla}_{\mathbf{D}, \mathbf{B}} \cdot(\mathbf{D} \odot \mathbf{E}+\mathbf{B} \odot \mathbf{H})=\partial_{j}\left(D^{j} \mathbf{E}+B^{j} \mathbf{H}\right), \\
& \mathbf{D} \nabla \mathbf{E}+\mathbf{B} \nabla \mathbf{H}=D^{j} \nabla E_{j}+B^{j} \nabla H_{j} .
\end{aligned}
$$

At this point, it is useful to introduce the Legendre transform of the electromagnetic part of the internal energy density $\ell_{u}^{\mathrm{em}}\left(s, n_{A}, \mathbf{E}, \mathbf{H}\right)$ defined as,

$$
\begin{aligned}
\ell_{u}^{\mathrm{em}}\left(s, n_{A}, \mathbf{E}, \mathbf{H}\right) \equiv & \frac{\partial u^{\mathrm{em}}}{\partial \mathbf{D}} \cdot \mathbf{D} \\
& +\frac{\partial u^{\mathrm{em}}}{\partial \mathbf{B}} \cdot \mathbf{B}-u^{\mathrm{em}}\left(s, n_{A}, \mathbf{D}, \mathbf{B}\right) .
\end{aligned}
$$

Using the constitutive equations (1), the expression (29) and the differential relations (31), the Legendre transform (49) is found to be,

$$
\ell_{u}^{\mathrm{em}}\left(s, n_{A}, \mathbf{E}, \mathbf{H}\right)=\frac{1}{2}(\mathbf{E} \cdot \mathbf{D}+\mathbf{H} \cdot \mathbf{B}),
$$

where,

$$
\begin{aligned}
& \mathbf{D}\left(s, n_{A}, \mathbf{E}\right)=\frac{\partial \ell_{u}^{\mathrm{em}}\left(s, n_{A}, \mathbf{E}, \mathbf{H}\right)}{\partial \mathbf{E}}, \\
& \mathbf{B}\left(s, n_{A}, \mathbf{H}\right)=\frac{\partial \ell_{u}^{\mathrm{em}}\left(s, n_{A}, \mathbf{E}, \mathbf{H}\right)}{\partial \mathbf{H}} .
\end{aligned}
$$

Using the constitutive equations (2), the Legendre transform (50) can be recast in terms of the electromagnetic fields $\mathbf{E}$ and $\mathbf{H}$ as,

$$
\begin{aligned}
\ell_{u}^{\mathrm{em}}\left(s, n_{A}, \mathbf{E}, \mathbf{H}\right)= & \frac{1}{2}\left(\varepsilon\left(s, n_{A}\right) \cdot(\mathbf{E} \odot \mathbf{E})\right. \\
& \left.+\boldsymbol{\mu}\left(s, n_{A}\right) \cdot(\mathbf{H} \odot \mathbf{H})\right),
\end{aligned}
$$

which establishes that

$$
\ell_{u}^{\mathrm{em}}\left(s, n_{A}, \mathbf{E}, \mathbf{H}\right)=u^{\mathrm{em}}\left(s, n_{A}, \mathbf{D}, \mathbf{B}\right) .
$$

The constitutive equations (2) imply that the gradient of the Legendre transform (52) is of the form,

$$
\begin{aligned}
\nabla \ell_{u}^{\mathrm{em}}= & \mathrm{D} \nabla \mathbf{E}+\mathbf{B} \nabla \mathbf{H} \\
& +\frac{1}{2}((\mathbf{E} \odot \mathbf{E}) \nabla \varepsilon+(\mathbf{H} \odot \mathbf{H}) \boldsymbol{\nabla} \boldsymbol{\mu}) .
\end{aligned}
$$


By substituting the relation (53) into the equation (48), the latter reduces to,

$$
\begin{aligned}
& \left(\nabla_{\mathbf{D}, \mathbf{B}} \cdot\left(\mathbf{D} \odot \mathbf{E}+\mathbf{B} \odot \mathbf{H}-\ell_{u}^{\mathrm{em}} \mathbb{1}\right)\right) \cdot \mathbf{v} \\
& =\left(q \mathbf{E}+\mathbf{j}_{q} \times \mathbf{B}-\frac{1}{2}((\mathbf{E} \odot \mathbf{E}) \nabla \varepsilon+(\mathbf{H} \odot \mathbf{H}) \boldsymbol{\nabla} \boldsymbol{\mu})\right) \cdot \mathbf{v} .
\end{aligned}
$$

By identification of the equations (45) and (54), the expressions for the electromagnetic stress tensor $\boldsymbol{\sigma}^{\mathrm{em}}$ and the electromagnetic force density $\mathbf{f}^{\mathrm{em}}$ are found to be,

$$
\begin{aligned}
\boldsymbol{\sigma}^{\mathrm{em}} & =\mathbf{D} \odot \mathbf{E}+\mathbf{B} \odot \mathbf{H}-\frac{1}{2}(\mathbf{E} \cdot \mathbf{D}+\mathbf{H} \cdot \mathbf{B}) \mathbb{1}, \\
\mathbf{f}^{\mathrm{em}} & =q \mathbf{E}+\mathbf{j}_{q} \times \mathbf{B}-\frac{1}{2}((\mathbf{E} \odot \mathbf{E}) \boldsymbol{\nabla} \boldsymbol{\varepsilon}+(\mathbf{H} \odot \mathbf{H}) \boldsymbol{\nabla} \boldsymbol{\mu}) .
\end{aligned}
$$

The first and second terms on the RHS of the expression (56) represent the Lorentz force density exerted by the electromagnetic fields $\mathbf{E}$ and $\mathbf{B}$ on the matter fields $q$ and $\mathbf{j}_{q}$. The last term represents the opposite of the ponderomotive force density exerted by the gradient of the matter fields $\boldsymbol{\varepsilon}\left(s, n_{A}\right)$ and $\boldsymbol{\mu}\left(s, n_{A}\right)$ on the electromagnetic fields $\mathbf{E}$ and $\mathbf{H}$. The Lorentz force density represents the action of the electromagnetic fields on the matter fields and the ponderomotive force density represents the reaction of the matter fields on the electromagnetic fields. Therefore, the ponderomotive force density has a negative sign in expression (56) for the electromagnetic force density $\mathbf{f}^{\mathrm{em}}$.

\subsubsection{Angular momentum continuity equation}

Since we do not include an intrinsic angular momentum in this publication, we shall refer to the orbital angular momentum simply as angular momentum. The angular momentum source density $\boldsymbol{\rho}_{\boldsymbol{\ell}}$ is due to the torque resulting from the action of the external force density $\mathbf{f}^{\mathrm{ext}}$, i.e.

$$
\boldsymbol{\rho}_{\ell}=\mathbf{r} \times \mathbf{f}^{\mathrm{ext}}
$$

where $\mathbf{r}$ is the position of the centre of mass of the local element of matter. The continuity equation for the angular momentum $\ell$ is given by,

$$
\dot{\ell}+(\nabla \cdot \mathbf{v}) \ell+\nabla \cdot j_{\ell}=\mathbf{r} \times \mathbf{f}^{\text {ext }},
$$

where the angular momentum density $\ell$ satisfies the dynamical relation,

$$
\ell=\mathbf{r} \times \mathbf{p} .
$$

From the definitions of the stress tensor (34) and the angular momentum density field (59), we deduce the expression of the orbital angular momentum current density tensor field $j_{\ell}$,

$$
j_{\ell}=\mathbf{r} \times j_{\mathbf{p}}=-\mathbf{r} \times \boldsymbol{\sigma} .
$$

Using relations (59) and (60), the continuity equation (58) becomes,

$$
\dot{\ell}+(\nabla \cdot \mathbf{v}) \ell-\nabla_{\boldsymbol{\sigma}} \cdot(\mathbf{r} \times \boldsymbol{\sigma})=\mathbf{r} \times \mathbf{f}^{\mathrm{ext}} .
$$

The vectorial product between the position $\mathbf{r}$ and the continuity equation for the momentum (33) yields,

$$
\mathbf{r} \times \dot{\mathbf{p}}+(\boldsymbol{\nabla} \cdot \mathbf{v}) \boldsymbol{\ell}-\mathbf{r} \times(\boldsymbol{\nabla} \cdot \boldsymbol{\sigma})=\mathbf{r} \times \mathbf{f}^{\mathrm{ext}}
$$

Using the vectorial identity,

$$
\dot{\ell}=\mathbf{v} \times \mathbf{p}+\mathbf{r} \times \dot{\mathbf{p}}
$$

where $\mathbf{v}=\dot{\mathbf{r}}$, the difference between the relations (62) and (61) is given by,

$$
\nabla_{\boldsymbol{\sigma}} \cdot(\mathbf{r} \times \boldsymbol{\sigma})-\mathbf{r} \times(\boldsymbol{\nabla} \cdot \boldsymbol{\sigma})-\mathbf{v} \times \mathbf{p}=\mathbf{0} .
$$

The definitions of the momentum densities (36)-(38) and the relation (3) imply that,

$$
\mathbf{v} \times \mathbf{p}=m \mathbf{v} \times \mathbf{v}+\frac{\mathbf{v}}{c} \times \frac{1}{c}\left[\left(\left(\mathbb{1}+\boldsymbol{\chi}_{e}\right) \cdot \mathbf{E}\right) \times\left(\left(\mathbb{1}+\boldsymbol{\chi}_{m}\right) \cdot \mathbf{H}\right)\right]
$$

is a relativistic correction proportional to $v / c$, which is neglected in a non-relativistic framework. Thus, in the nonrelativistic limit, the relation (63) reduces to,

$$
\nabla_{\sigma} \cdot(\mathbf{r} \times \boldsymbol{\sigma})-\mathbf{r} \times(\nabla \cdot \sigma)=\mathbf{0}
$$

where the vectorial terms on the LHS of the relation (64) are written explicitly in components as,

$$
\begin{aligned}
\left(\boldsymbol{\nabla}_{\boldsymbol{\sigma}} \cdot(\mathbf{r} \times \boldsymbol{\sigma})\right)^{i} & =\varepsilon^{i j k} \partial_{l}\left(r_{j} \sigma_{k}^{l}\right) \\
& =\varepsilon^{i j k}\left(\sigma_{j k}+r_{j} \partial_{l} \sigma_{k}^{l}\right), \\
(\mathbf{r} \times(\boldsymbol{\nabla} \cdot \boldsymbol{\sigma}))^{i} & =\varepsilon^{i j k} r_{j} \partial_{l} \sigma_{k}^{l},
\end{aligned}
$$

using the Einstein implicit summation convention, the components of the totally antisymmetric contravariant Levi-Civita tensor $\varepsilon^{i j k}$ and the trivial identity $\partial_{i} r_{j}=\delta_{i j}$. Hence, the relation (64) can be recast as,

$$
\hat{\sigma}=\mathbf{0}
$$

where $\hat{\boldsymbol{\sigma}}$ is the stress pseudo-vector defined as the dual of the antisymmetric part of the stress tensor and written explicitly in components as,

$$
\hat{\boldsymbol{\sigma}}^{i}=\varepsilon^{i j k} \sigma_{j k} .
$$

Thus, the stress tensor $\boldsymbol{\sigma}$ is a symmetric rank-2 tensor.

\subsubsection{Internal energy continuity equation}

The continuity equation for the internal energy density $u$ reads,

$$
\dot{u}+(\nabla \cdot \mathbf{v}) u+\nabla \cdot \mathbf{j}_{u}=\rho_{u} .
$$

Taking the time derivative of the energy (27) and using the continuity equations for the mass (43), the energy (21) and the internal energy (66), the second law of Newton (44), the electromagnetic dynamical equation (45), the splitting (39) and the vectorial identity,

$$
(\boldsymbol{\nabla} \cdot \boldsymbol{\sigma}) \cdot \mathbf{v}=\nabla_{\boldsymbol{\sigma}} \cdot(\boldsymbol{\sigma} \cdot \mathbf{v})-\boldsymbol{\sigma} \cdot(\boldsymbol{\nabla} \odot \mathbf{v})
$$


the expression for the internal energy current density $\mathbf{j}_{u}$ and the internal energy source density $\rho_{u}$ are identified respectively as,

$$
\begin{aligned}
\mathbf{j}_{u} & =\mathbf{j}_{e}+\boldsymbol{\sigma} \cdot \mathbf{v}, \\
\rho_{u} & =\boldsymbol{\sigma} \cdot(\boldsymbol{\nabla} \odot \mathbf{v}) .
\end{aligned}
$$

Note that,

$$
\mathbf{j}_{W}=-\boldsymbol{\sigma} \cdot \mathbf{v},
$$

represents the work current density due to the matter and electromagnetic fields.

The physical interpretation of the expression (68) for the source density $\rho_{u}$ is also clear. The source of internal energy density is due to the shear on the local element of matter $d \Sigma$. It is important to emphasize that even in the local material frame where the local center of mass has no velocity, i.e. $\mathbf{v}=\mathbf{0}$, the infinitesimal element of matter is still subjected to the constraints due to the environment, which generate deformations $(\boldsymbol{\nabla} \odot \mathbf{v})$.

\section{Evolution equations}

\subsection{Conservation of the mass}

The mass density field $m$ is a property of the matter and thus a function of the matter state fields, i.e. $m=m\left(s, n_{A}\right)$. The time derivative of the mass density field yields,

$$
\dot{m}=\frac{\partial m}{\partial s} \dot{s}+\sum_{A} \frac{\partial m}{\partial n_{A}} \dot{n}_{A}
$$

Using this relation and the continuity equations for the entropy (7) and the chemical substance $A(9)$, the continuity equation for the mass (43) is recast as,

$$
\begin{aligned}
& \frac{\partial m}{\partial s}\left(\rho_{s}-(\boldsymbol{\nabla} \cdot \mathbf{v}) s-\boldsymbol{\nabla} \cdot \mathbf{j}_{s}\right)+(\boldsymbol{\nabla} \cdot \mathbf{v}) m \\
& +\sum_{A} \frac{\partial m}{\partial n_{A}}\left(\rho_{A}-(\boldsymbol{\nabla} \cdot \mathbf{v}) n_{A}-\boldsymbol{\nabla} \cdot \mathbf{j}_{A}\right)=0 .
\end{aligned}
$$

The evolution condition of the second law requires the entropy production to be positive, i.e. $\rho_{s} \geqslant 0$, irrespective of the value and sign of the other fields. Thus, the partial derivative factor multiplying $\rho_{s}$ has to vanish, i.e.

$$
\frac{\partial m}{\partial s}=0 .
$$

This implies that the mass density is only a function of the densities $n_{A}$, i.e. $m=m\left(n_{A}\right)$. Hence, the equation (71) reduces to,

$$
\left(m-\sum_{A} \frac{\partial m}{\partial n_{A}} n_{A}\right)(\boldsymbol{\nabla} \cdot \mathbf{v})+\sum_{A} \frac{\partial m}{\partial n_{A}}\left(\rho_{A}-\boldsymbol{\nabla} \cdot \mathbf{j}_{A}\right)=0 .
$$

The relation (73) has to hold locally for all matter flows, which implies that the terms in the first brackets have to vanish according to,

$$
m-\sum_{A} \frac{\partial m}{\partial n_{A}} n_{A}=0 .
$$

The derivative of relation (74) with respect to the density $n_{B}$ of a chemical substance $B$ yields,

$$
\frac{\partial m}{\partial n_{B}}-\sum_{A}\left(\frac{\partial^{2} m}{\partial n_{A} \partial n_{B}} n_{A}+\frac{\partial m}{\partial n_{A}} \delta_{A B}\right)=0
$$

which implies that,

$$
\frac{\partial^{2} m}{\partial n_{A} \partial n_{B}}=0 \quad \forall A,
$$

and therefore the partial derivatives,

$$
m_{A} \equiv \frac{\partial m}{\partial n_{A}}=\mathrm{cst},
$$

are independent constants representing the mass of a unit of chemical substance $A$. Thus, the mass density field $m$ is the sum of the mass densities of the different chemical substances according to,

$$
m=\sum_{A} m_{A} n_{A} .
$$

From the relations (73) and (74), it follows that,

$$
\sum_{A} m_{A}\left(\rho_{A}-\nabla \cdot \mathbf{j}_{A}\right)=0 .
$$

Using the fact that the mass $m_{A}$ is an invariant, i.e.

$$
\boldsymbol{\nabla} m_{A}=\mathbf{0},
$$

which is a consequence of (77), the equation (79) can be recast as,

$$
\sum_{A} m_{A} \rho_{A}-\nabla \cdot\left(\sum_{A} m_{A} \mathbf{j}_{A}\right)=0 .
$$

It has to hold in the presence or in the absence of chemical reactions, i.e.

$$
\sum_{A} \rho_{A} m_{A}=0,
$$

which is expressed in terms of the stoichiometric coefficients $\nu_{a A}$ using the expression (8) according to,

$$
\sum_{A} m_{A}\left(\sum_{a} \omega_{a} \nu_{a A}\right)=0 .
$$

Since the reaction rates $\omega_{a}$ are independent, each chemical reaction $a$ satisfies the mass conservation law,

$$
\sum_{A} m_{A} \nu_{a A}=0,
$$

which is the law of Lavoisier. 
Substituting condition (82) into equation (81), the latter reduces to,

$$
\nabla \cdot\left(\sum_{A} m_{A} \mathbf{j}_{A}\right)=0
$$

It has to hold for any current flow, which defines the material frame where the local center of mass of the matter element is at rest, i.e.

$$
\sum_{A} m_{A} \mathbf{j}_{A}=\mathbf{0}
$$

This condition allows for a coherent physical interpretation of the velocity $\mathbf{v}$, as we now show. We define the velocity $\mathbf{v}_{A}$ of the substance $A$ as,

$$
\mathbf{v}_{A} n_{A} \equiv \mathbf{v} n_{A}+\mathbf{j}_{A} .
$$

Using relations $(37),(78),(86)$ and $(87)$, the matter momentum density $\mathbf{p}^{\text {mat }}$ becomes,

$$
\mathbf{p}^{\text {mat }}=\sum_{A} m_{A} n_{A} \mathbf{v}_{A}
$$

Thus, the velocity $\mathbf{v}$ is found to be,

$$
\mathbf{v}=\frac{\sum_{A} m_{A} n_{A} \mathbf{v}_{A}}{\sum_{A} m_{A} n_{A}}
$$

and represents the velocity of the center of mass of the infinitesimal mass element $d m$ contained within the local infinitesimal thermodynamic system $d \Sigma$. It is the barycentric velocity of the local element of matter.

\subsection{Conservation of the electric charge}

The electric charge density field $q$ is a property of the matter and thus a function of the matter state fields, i.e. $q=q\left(s, n_{A}\right)$. The time derivative of the electric charge density field $q$ yields,

$$
\dot{q}=\frac{\partial q}{\partial s} \dot{s}+\sum_{A} \frac{\partial q}{\partial n_{A}} \dot{n}_{A} .
$$

Using this relation and the continuity equations for the entropy (7) and the chemical substance $A(9)$, the continuity equation for the charge (4) is recast as,

$$
\begin{aligned}
& \frac{\partial q}{\partial s}\left(\rho_{s}-(\boldsymbol{\nabla} \cdot \mathbf{v}) s-\boldsymbol{\nabla} \cdot \mathbf{j}_{s}\right)+(\boldsymbol{\nabla} \cdot \mathbf{v}) q+\nabla \cdot \mathbf{j}_{q} \\
& +\sum_{A} \frac{\partial q}{\partial n_{A}}\left(\rho_{A}-(\boldsymbol{\nabla} \cdot \mathbf{v}) n_{A}-\boldsymbol{\nabla} \cdot \mathbf{j}_{A}\right)=0
\end{aligned}
$$

In a similar way to the mass density, the charge density cannot be a function of the entropy in order to satisfy the evolution condition of the second law. Hence, the electric charge density is only a function of the densities $n_{A}$, i.e. $q=q\left(n_{A}\right)$. Hence, equation (91) reduces to,

$$
\begin{aligned}
& \left(q-\sum_{A} \frac{\partial q}{\partial n_{A}} n_{A}\right)(\boldsymbol{\nabla} \cdot \mathbf{v}) \\
& +\sum_{A} \frac{\partial q}{\partial n_{A}}\left(\rho_{A}-\nabla \cdot \mathbf{j}_{A}\right)+\nabla \cdot \mathbf{j}_{q}=0 .
\end{aligned}
$$

The relation (92) has to hold locally for all matter flows, which implies that the terms in the first brackets have to vanish according to,

$$
q-\sum_{A} \frac{\partial q}{\partial n_{A}} n_{A}=0
$$

The derivative of the relation (93) with respect to the density $n_{B}$ of a chemical substance $B$ yields,

$$
\frac{\partial q}{\partial n_{B}}-\sum_{A}\left(\frac{\partial^{2} q}{\partial n_{A} \partial n_{B}} n_{A}+\frac{\partial q}{\partial n_{A}} \delta_{A B}\right)=0
$$

which implies that,

$$
\frac{\partial^{2} q}{\partial n_{A} \partial n_{B}}=0 \quad \forall A,
$$

and therefore the partial derivatives,

$$
q_{A} \equiv \frac{\partial q}{\partial n_{A}}=\mathrm{cst}
$$

are independent constants representing the free electric charge of a unit of chemical substance $A$. Thus, the electric charge density field $q_{A}$ is the sum of the electric charge densities of the different chemical substances according to,

$$
q=\sum_{A} q_{A} n_{A}
$$

From the relations (92) and (93), it follows that,

$$
\sum_{A} q_{A}\left(\rho_{A}-\nabla \cdot \mathbf{j}_{A}\right)+\nabla \cdot \mathbf{j}_{q}=0
$$

Using the fact that the electric charge $q_{A}$ is an invariant, i.e.

$$
\nabla q_{A}=\mathbf{0}
$$

which is a consequence of (96), the equation (98) can be recast as,

$$
\sum_{A} q_{A} \rho_{A}+\nabla \cdot\left(\mathbf{j}_{q}-\sum_{A} m_{A} \mathbf{j}_{A}\right)=0 .
$$

It has to hold in the presence or in the absence of chemical reactions, i.e.

$$
\sum_{A} \rho_{A} q_{A}=0
$$


which is expressed in terms of the stoichiometric coefficients $\nu_{a A}$ using the expression (8) according to,

$$
\sum_{A} q_{A}\left(\sum_{a} \omega_{a} \nu_{a A}\right)=0
$$

Since the reaction rates $\omega_{a}$ are independent, each chemical reaction $a$ satisfies the charge conservation law,

$$
\sum_{A} q_{A} \nu_{a A}=0
$$

Substituting the condition (101) into the equation (100), the latter reduces to,

$$
\nabla \cdot\left(\mathbf{j}_{q}-\sum_{A} q_{A} \mathbf{j}_{A}\right)=0 .
$$

It has to hold for any current flow, which yields an expression for the diffusive electric current density $\mathbf{j}_{q}$ in terms of the chemical composition, i.e.

$$
\mathbf{j}_{q}=\sum_{A} q_{A} \mathbf{j}_{A}
$$

Using the relations (87), (97) and (105), the expression (5) for the electric current density in the spatial frame becomes,

$$
\mathbf{j}_{q}^{\prime}=\sum_{A} q_{A} n_{A} \mathbf{v}_{A}
$$

There are four types of electric charge carriers in our model, which are considered as different types of chemical substances $A$ of the matter:

- free electrons (i.e. $A=e$ ), $q_{e}<0$,

- anions, $q_{A}<0$,

- cations, $q_{A}>0$,

- neutral substances, $q_{A}=0$.

Note that for physical consistency, free electrons are included as a separate chemical substance in our phenomenological model.

\subsection{Matter dynamics}

In order to characterise physically the matter dynamics, we substitute the expression (88) for the chemical nature of the matter momentum density and the continuity equation of the chemical substance (9) into the continuity equation (40) for the matter momentum density $\mathbf{p}^{\text {mat }}$. The latter then becomes,

$$
\begin{aligned}
& \sum_{A}\left(n_{A} m_{A} \dot{\mathbf{v}}_{A}-\left(\boldsymbol{\nabla} \cdot \mathbf{j}_{A}\right) m_{A} \mathbf{v}_{A}+\rho_{A} m_{A} \mathbf{v}_{A}\right) \\
& -\boldsymbol{\nabla} \cdot \boldsymbol{\sigma}^{\mathrm{mat}}=\mathbf{f}^{\mathrm{ext}}+\mathbf{f}^{\mathrm{em}} .
\end{aligned}
$$

Using the vectorial identity,

$$
\left(\boldsymbol{\nabla} \cdot \mathbf{j}_{A}\right) m_{A} \mathbf{v}_{A}=\boldsymbol{\nabla}_{\mathbf{j}} \cdot\left(m_{A} \mathbf{v}_{A} \odot \mathbf{j}_{A}\right)-\left(\mathbf{j}_{A} \cdot \boldsymbol{\nabla}\right) m_{A} \mathbf{v}_{A},
$$

where the index $\mathbf{j}$ denotes that there is a dot product between the covariant differential operator $\nabla$ and the contravariant current density $\mathbf{j}_{A}$, the continuity equation (107) for the matter momentum density $\mathbf{p}^{\text {mat }}$ can be recast as,

$$
\begin{aligned}
& \sum_{A} m_{A}\left(n_{A} \dot{\mathbf{v}}_{A}+\left(\mathbf{j}_{A} \cdot \nabla\right) \mathbf{v}_{A}\right)+\sum_{A} \rho_{A} m_{A} \mathbf{v}_{A} \\
& -\nabla_{\mathbf{j}} \cdot\left(\boldsymbol{\sigma}^{\mathrm{mat}}+\sum_{A} m_{A} \mathbf{v}_{A} \odot \mathbf{j}_{A}\right)=\mathbf{f}^{\mathrm{ext}}+\mathbf{f}^{\mathrm{em}} .
\end{aligned}
$$

The matter stress tensor $\boldsymbol{\sigma}^{\text {mat }}$ is split into a reversible part due to the pressure $P$ and an irreversible part $\tilde{\sigma}^{\text {mat }}$. The pressure $P$ is isotropic in the local material frame and it opposes the constraints on the matter $\boldsymbol{\sigma}^{\text {mat }}$, i.e.

$$
\boldsymbol{\sigma}^{\mathrm{mat}}=-P \mathbb{1}+\tilde{\boldsymbol{\sigma}}^{\mathrm{mat}},
$$

which implies that the continuity equation (108) is recast as,

$$
\begin{aligned}
& \sum_{A} m_{A}\left(n_{A} \dot{\mathbf{v}}_{A}+\left(\mathbf{j}_{A} \cdot \nabla\right) \mathbf{v}_{A}\right)+\nabla P+\sum_{A} \rho_{A} m_{A} \mathbf{v}_{A} \\
& -\nabla_{\mathbf{j}} \cdot\left(\tilde{\boldsymbol{\sigma}}^{\mathrm{mat}}+\sum_{A} m_{A} \mathbf{v}_{A} \odot \mathbf{j}_{A}\right)=\mathbf{f}^{\mathrm{ext}}+\mathbf{f}^{\mathrm{em}} .
\end{aligned}
$$

It has to hold in the presence or in the absence of chemical reactions, i.e.

$$
\sum_{A} \rho_{A} m_{A} \mathbf{v}_{A}=\mathbf{0}
$$

which means that the chemical reactions preserve the local momentum of the matter. Moreover, it has to hold for any current flow, which yields an explicit expression for the irreversible part of the matter stress tensor, i.e.

$$
\tilde{\boldsymbol{\sigma}}^{\text {mat }}=-\sum_{A} m_{A} \mathbf{v}_{A} \odot \mathbf{j}_{A}
$$

The conditions (111) and (112) imply that the continuity equation (110) reduces to,

$$
\sum_{A} m_{A}\left(n_{A} \dot{\mathbf{v}}_{A}+\left(\mathbf{j}_{A} \cdot \nabla\right) \mathbf{v}_{A}\right)=\mathbf{f}^{\mathrm{ext}}+\mathbf{f}^{\mathrm{em}}-\nabla P,
$$

which describes the matter dynamics that is contained in the time evolution of the chemical substances.

\subsection{Thermostatic equation, reversible and irreversible thermodynamic equations}

In order to determine the internal energy balance, we take the time derivative of the internal energy density field $u\left(s, n_{A}, \mathbf{D}, \mathbf{B}\right)$ of the system $\Sigma$, i.e.

$$
\dot{u}=\frac{\partial u}{\partial s} \dot{s}+\sum_{A} \frac{\partial u}{\partial n_{A}} \dot{n}_{A}+\frac{\partial u}{\partial \mathbf{D}} \cdot \dot{\mathbf{D}}+\frac{\partial u}{\partial \mathbf{B}} \cdot \dot{\mathbf{B}} .
$$

The local thermodynamic system $d \Sigma$ is assumed to be at equilibrium. Thus, we can define the intensive 
fields temperature $T\left(s, n_{A}, \mathbf{D}, \mathbf{B}\right)$ and chemical potential $\mu_{A}\left(s, n_{A}, \mathbf{D}, \mathbf{B}\right)$ of the substance $A$ as the conjugate of the state scalar fields densities $s$ and $n_{A}$ respectively, i.e.

$$
\begin{gathered}
T\left(s, n_{A}, \mathbf{D}, \mathbf{B}\right) \equiv \frac{\partial u\left(s, n_{A}, \mathbf{D}, \mathbf{B}\right)}{\partial s}, \\
\mu_{A}\left(s, n_{A}, \mathbf{D}, \mathbf{B}\right) \equiv \frac{\partial u\left(s, n_{A}, \mathbf{D}, \mathbf{B}\right)}{\partial n_{A}} .
\end{gathered}
$$

In order to determine the temperature of the system in terms of the electromagnetic fields, we explicitly substitute the expressions (28) and (29) for the internal energy density $u\left(s, n_{A}, \mathbf{D}, \mathbf{B}\right)$ into the definition of the temperature (115) using the vectorial identities,

$$
\begin{gathered}
\frac{\partial \varepsilon^{-1}}{\partial s}=-\frac{\partial \varepsilon}{\partial s} \cdot \varepsilon^{-2} \\
\frac{\partial \boldsymbol{\mu}^{-1}}{\partial s}=-\frac{\partial \boldsymbol{\mu}}{\partial s} \cdot \boldsymbol{\mu}^{-2}
\end{gathered}
$$

to obtain,

$$
\begin{aligned}
T\left(s, n_{A}, \mathbf{D}, \mathbf{B}\right)= & T\left(s, n_{A}, \mathbf{0}, \mathbf{0}\right)-\frac{1}{2}\left(\frac{\partial \varepsilon}{\partial s} \cdot \varepsilon^{-2} \cdot(\mathbf{D} \odot \mathbf{D})\right. \\
& \left.+\frac{\partial \boldsymbol{\mu}}{\partial s} \cdot \boldsymbol{\mu}^{-2} \cdot(\mathbf{B} \odot \mathbf{B})\right) .
\end{aligned}
$$

Using the linear electromagnetic constitutive equations (1), the expression for the temperature (116) is recast as,

$$
\begin{aligned}
T\left(s, n_{A}, \mathbf{D}, \mathbf{B}\right)= & T\left(s, n_{A}, \mathbf{0}, \mathbf{0}\right)-\frac{1}{2}\left(\frac{\partial \varepsilon}{\partial s} \cdot(\mathbf{E} \odot \mathbf{E})\right. \\
& \left.+\frac{\partial \boldsymbol{\mu}}{\partial s} \cdot(\mathbf{H} \odot \mathbf{H})\right) .
\end{aligned}
$$

Note that the phenomenological tensors $\partial \varepsilon / \partial s$ and $\partial \boldsymbol{\mu} / \partial s$ are closely related to the temperature dependence of the electric permittivity tensor and the magnetic permeability tensor, respectively.

In order to determine the chemical potential of the system in terms of the electromagnetic fields, we explicitly substitute the expressions (28) and (29) for the internal energy density $u\left(s, n_{A}, \mathbf{D}, \mathbf{B}\right)$ into the definition of the chemical potential of the substance A (115) using the vectorial identities,

$$
\begin{aligned}
& \frac{\partial \varepsilon^{-1}}{\partial n_{A}}=-\frac{\partial \varepsilon}{\partial n_{A}} \cdot \varepsilon^{-2}, \\
& \frac{\partial \boldsymbol{\mu}^{-1}}{\partial n_{A}}=-\frac{\partial \boldsymbol{\mu}}{\partial n_{A}} \cdot \boldsymbol{\mu}^{-2}
\end{aligned}
$$

to obtain,

$$
\begin{aligned}
\mu_{A}\left(s, n_{A}, \mathbf{D}, \mathbf{B}\right)= & \mu_{A}\left(s, n_{A}, \mathbf{0}, \mathbf{0}\right)-\frac{1}{2}\left(\frac{\partial \varepsilon}{\partial n_{A}} \cdot \varepsilon^{-2} \cdot(\mathbf{D} \odot \mathbf{D})\right. \\
& \left.+\frac{\partial \boldsymbol{\mu}}{\partial n_{A}} \cdot \boldsymbol{\mu}^{-2} \cdot(\mathbf{B} \odot \mathbf{B})\right) .
\end{aligned}
$$

Using the linear electromagnetic constitutive equations (1), the expression for the chemical potential of the substance A (118) is recast as,

$$
\begin{aligned}
\mu_{A}\left(s, n_{A}, \mathbf{D}, \mathbf{B}\right)= & \mu_{A}\left(s, n_{A}, \mathbf{0}, \mathbf{0}\right) \\
& -\frac{1}{2}\left(\frac{\partial \varepsilon}{\partial n_{A}} \cdot(\mathbf{E} \odot \mathbf{E})+\frac{\partial \boldsymbol{\mu}}{\partial n_{A}} \cdot(\mathbf{H} \odot \mathbf{H})\right) .
\end{aligned}
$$

Note that the phenomenological tensors $\partial \varepsilon / \partial n_{A}$ and $\partial \boldsymbol{\mu} / \partial n_{A}$ describe, respectively, the dependence of the electric permittivity tensor and the magnetic permeability tensor on the density of the chemical substance $A$.

Using the relations (31) and (115), the time derivative of the internal energy density (114) is recast as,

$$
\dot{u}=T \dot{s}+\sum_{A} \mu_{A} \dot{n}_{A}+\mathbf{E} \cdot \dot{\mathbf{D}}+\mathbf{H} \cdot \dot{\mathbf{B}} .
$$

The first law or energy balance is given by the continuity equation for the internal energy (66). Using the continuity equations for the entropy (7) and the chemical substance $A$ (9), Maxwell-Ampère's law (14), Faraday's law (19) and the internal energy source density (68), the continuity equation for the internal energy (66) is recast as,

$$
\begin{aligned}
& T\left(\rho_{s}-(\boldsymbol{\nabla} \cdot \mathbf{v}) s-\boldsymbol{\nabla} \cdot \mathbf{j}_{s}\right) \\
& +\sum_{A} \mu_{A}\left(\rho_{A}-(\boldsymbol{\nabla} \cdot \mathbf{v}) n_{A}-\boldsymbol{\nabla} \cdot \mathbf{j}_{A}\right) \\
& +\mathbf{E} \cdot\left(\boldsymbol{\nabla} \times \mathbf{H}-(\boldsymbol{\nabla} \cdot \mathbf{v}) \mathbf{D}+(\mathbf{D} \cdot \boldsymbol{\nabla}) \mathbf{v}-\mathbf{j}_{q}\right) \\
& +\mathbf{H} \cdot(-\boldsymbol{\nabla} \times \mathbf{E}-(\boldsymbol{\nabla} \cdot \mathbf{v}) \mathbf{B}+(\mathbf{B} \cdot \boldsymbol{\nabla}) \mathbf{v}) \\
& +(\boldsymbol{\nabla} \cdot \mathbf{v}) u+\boldsymbol{\nabla} \cdot \mathbf{j}_{u}=\boldsymbol{\sigma} \cdot(\boldsymbol{\nabla} \odot \mathbf{v}) .
\end{aligned}
$$

Moreover, using the vectorial identities,

$$
\begin{aligned}
& T\left(\boldsymbol{\nabla} \cdot \mathbf{j}_{s}\right)=\nabla \cdot\left(T \mathbf{j}_{s}\right)-\mathbf{j}_{s} \cdot \nabla T, \\
& \sum_{A} \mu_{A}\left(\boldsymbol{\nabla} \cdot \mathbf{j}_{A}\right)=\nabla \cdot\left(\sum_{A} \mu_{A} \mathbf{j}_{A}\right)-\sum_{A} \mathbf{j}_{A} \cdot \nabla \mu_{A}, \\
& \mathbf{H} \cdot(\boldsymbol{\nabla} \times \mathbf{E})-\mathbf{E} \cdot(\boldsymbol{\nabla} \times \mathbf{H})=\nabla \cdot(\mathbf{E} \times \mathbf{H}),
\end{aligned}
$$

the internal energy balance equation (121) becomes,

$$
\begin{aligned}
& \left(u-T s-\sum_{A} \mu_{A} n_{A}-\mathbf{E} \cdot \mathbf{D}-\mathbf{H} \cdot \mathbf{B}\right)(\boldsymbol{\nabla} \cdot \mathbf{v}) \\
& +\nabla \cdot\left(\mathbf{j}_{u}-T \mathbf{j}_{s}-\sum_{A} \mu_{A} \mathbf{j}_{A}-\mathbf{E} \times \mathbf{H}\right) \\
& +T \rho_{s}+\sum_{A} \mu_{A} \rho_{A}-\boldsymbol{\sigma} \cdot(\boldsymbol{\nabla} \odot \mathbf{v}) \\
& +\mathbf{j}_{s} \cdot \nabla T+\sum_{A} \mathbf{j}_{A} \cdot \nabla \mu_{A}-\mathbf{j}_{q} \cdot \mathbf{E} \\
& +(\mathbf{E}(\mathbf{D} \cdot \nabla)+\mathbf{H}(\mathbf{B} \cdot \nabla)) \cdot \mathbf{v}=0 .
\end{aligned}
$$


It is useful to introduce the chemical affinity $\mathcal{A}_{a}$ of a chemical reaction $a$ defined as,

$$
\mathcal{A}_{a}=-\sum_{A} \nu_{a A} \mu_{A} .
$$

Expressions (8) and (123) imply that the sum over the chemical substances $A$ can be replaced by a sum over the chemical reactions $a$ according to,

$$
\sum_{A} \mu_{A} \rho_{A}=-\sum_{a} \omega_{a} \mathcal{A}_{a} .
$$

Moreover, using the definition (55) of the electromagnetic stress tensor $\boldsymbol{\sigma}^{\mathrm{em}}$ and the splitting (39), the vectorial identity,

$$
\begin{aligned}
& (\mathbf{E}(\mathbf{D} \cdot \boldsymbol{\nabla})+\mathbf{H}(\mathbf{B} \cdot \boldsymbol{\nabla})) \cdot \mathbf{v}= \\
& (\mathbf{D} \odot \mathbf{E}+\mathbf{B} \odot \mathbf{H}) \cdot(\boldsymbol{\nabla} \odot \mathbf{v}),
\end{aligned}
$$

is recast as,

$$
\begin{aligned}
(\mathbf{E}(\mathbf{D} \cdot \boldsymbol{\nabla})+\mathbf{H}(\mathbf{B} \cdot \boldsymbol{\nabla})) \cdot \mathbf{v}= & \left(\boldsymbol{\sigma}-\boldsymbol{\sigma}^{\mathrm{mat}}\right) \cdot(\boldsymbol{\nabla} \odot \mathbf{v}) \\
& +\frac{1}{2}(\mathbf{E} \cdot \mathbf{D}+\mathbf{H} \cdot \mathbf{B})(\boldsymbol{\nabla} \cdot \mathbf{v}) .
\end{aligned}
$$

Using the identities (109) and (124), the internal energy balance equation (122) becomes,

$$
\begin{aligned}
& \left(u-T s+P-\sum_{A} \mu_{A} n_{A}-\frac{1}{2}(\mathbf{E} \cdot \mathbf{D}+\mathbf{H} \cdot \mathbf{B})\right)(\boldsymbol{\nabla} \cdot \mathbf{v}) \\
& +\boldsymbol{\nabla} \cdot\left(\mathbf{j}_{u}-T \mathbf{j}_{s}-\sum_{A} \mu_{A} \mathbf{j}_{A}-\mathbf{E} \times \mathbf{H}\right) \\
& +T \rho_{s}-\sum_{a} \omega_{a} \mathcal{A}_{a}-\tilde{\boldsymbol{\sigma}}^{\mathrm{mat}} \cdot(\boldsymbol{\nabla} \odot \mathbf{v}) \\
& +\mathbf{j}_{s} \cdot \boldsymbol{\nabla} T+\sum_{A} \mathbf{j}_{A} \cdot \boldsymbol{\nabla} \mu_{A}-\mathbf{j}_{q} \cdot \mathbf{E}=0 .
\end{aligned}
$$

Using expressions (105) and (112) for the diffusive electric current density vector and the matter momentum stress tensor, we obtain the identities,

$$
\begin{aligned}
& \mathbf{j}_{q} \cdot \mathbf{E}=\sum_{A} q_{A} \mathbf{j}_{A} \cdot \mathbf{E}, \\
& \tilde{\boldsymbol{\sigma}}^{\mathrm{mat}} \cdot(\boldsymbol{\nabla} \odot \mathbf{v})=\sum_{A} \mathbf{j}_{A} \cdot\left(-m_{A} \mathbf{v}_{A} \boldsymbol{\nabla} \mathbf{v}\right) .
\end{aligned}
$$

Using the identities (127), the internal energy balance equation (126) becomes,

$$
\begin{aligned}
& \left(u-T s+P-\sum_{A} \mu_{A} n_{A}-\frac{1}{2}(\mathbf{E} \cdot \mathbf{D}+\mathbf{H} \cdot \mathbf{B})\right)(\boldsymbol{\nabla} \cdot \mathbf{v}) \\
& +\boldsymbol{\nabla} \cdot\left(\mathbf{j}_{u}-T \mathbf{j}_{s}-\sum_{A} \mu_{A} \mathbf{j}_{A}-\mathbf{E} \times \mathbf{H}\right) \\
& +T \rho_{s}-\sum_{a} \omega_{a} \mathcal{A}_{a}-\mathbf{j}_{s} \cdot(-\boldsymbol{\nabla} T) \\
& -\sum_{A} \mathbf{j}_{A} \cdot\left(-\nabla \mu_{A}+q_{A} \mathbf{E}-m_{A} \mathbf{v}_{A} \boldsymbol{\nabla} \mathbf{v}\right)=0 .
\end{aligned}
$$

The internal energy balance equation (128) has to hold locally for all flows. This implies that the terms in the first brackets have to vanish, which yields the thermostatic equilibrium equation for the matter, i.e.

$$
u=T s-P+\sum_{A} \mu_{A} n_{A}+\frac{1}{2}(\mathbf{E} \cdot \mathbf{D}+\mathbf{H} \cdot \mathbf{B}) .
$$

Similarly, the internal energy balance equation (128) has to hold locally for all currents. This implies that the terms in the second brackets have to vanish, which yields the reversible thermodynamic evolution equation for the matter, i.e.

$$
\mathbf{j}_{u}=T \mathbf{j}_{s}+\sum_{A} \mu_{A} \mathbf{j}_{A}+\mathbf{E} \times \mathbf{H} .
$$

The mechanical work current density $\mathbf{j}_{W}$, the heat current density $\mathbf{j}_{Q}$, the chemical energy current density $\mathbf{j}_{\mathrm{CH}}$ and the electromagnetic energy current density $\mathbf{j}_{\mathrm{EM}}$ satisfy,

$$
\mathbf{j}_{e}=\mathbf{j}_{W}+\mathbf{j}_{u}=\mathbf{j}_{W}+\mathbf{j}_{Q}+\mathbf{j}_{\mathrm{CH}}+\mathbf{j}_{\mathrm{EM}},
$$

and are respectively found to be,

$$
\begin{aligned}
\mathbf{j}_{W} & =-\mathbf{v} \cdot \boldsymbol{\sigma}=\mathbf{v} \cdot j_{\mathbf{p}}, \\
\mathbf{j}_{Q} & =T \mathbf{j}_{s}, \\
\mathbf{j}_{\mathrm{CH}} & =\sum_{A} \mu_{A} \mathbf{j}_{A}, \\
\mathbf{j}_{\mathrm{EM}} & =\mathbf{E} \times \mathbf{H} .
\end{aligned}
$$

Finally, the thermostatic equilibrium equation (129) and the reversible thermodynamic evolution equation (130) imply that the internal energy balance equation (128) yields the irreversible thermodynamic evolution equation for the matter, i.e.

$$
\begin{aligned}
\rho_{s}= & \frac{1}{T}\left\{\sum_{a} \omega_{a} \mathcal{A}_{a}+\mathbf{j}_{s} \cdot(-\nabla T)\right. \\
& \left.+\sum_{A} \mathbf{j}_{A} \cdot\left(-\nabla \mu_{A}+q_{A} \mathbf{E}-m_{A} \mathbf{v}_{A} \boldsymbol{\nabla} \mathbf{v}\right)\right\} .
\end{aligned}
$$

\section{Thermodynamical phenomenology}

\subsection{Linear phenomenological relations and Onsager matrix elements}

In order to deduce the linear phenomenological relations, we follow the approach developed by Onsager $[14,15]$. Expression (133) can be formally split into a scalar and a vectorial part, which are irreducible representations of the Euclidean group and have different symmetries. Thus, the entropy source density (133) is expressed formally as,

$$
\rho_{s}=\frac{1}{T}\left\{\sum_{a} \omega_{a} \mathcal{A}_{a}+\sum_{\alpha} \mathbf{j}_{\alpha} \cdot \mathbf{F}_{\alpha}\right\},
$$


where $\omega_{a}$ is a scalar chemical reaction rate density, $\mathcal{A}_{a}$ is a scalar chemical affinity, $\mathbf{j}_{\alpha}$ is a vectorial current density, $\mathbf{F}_{\alpha}$ is a vectorial force.

In relation (134), there are two types of vectorial current densities and forces (i.e. $\alpha \in\{s, A\}$ ). First, there is a thermal current density $\mathbf{j}_{s}$ and a dissipative thermal force $\mathbf{F}_{s}=-\boldsymbol{\nabla} T$. Second, there are current densities $\mathbf{j}_{A}$ for the chemical substances $A$ and dissipative forces $\mathbf{F}_{A}$ acting on these substances. The expression for the forces $\mathbf{F}_{A}$ is given by the dissipation relation (133), i.e.

$$
\mathbf{F}_{A}=-\nabla \mu_{A}+q_{A} \mathbf{E}-m_{A} \mathbf{v}_{A} \boldsymbol{\nabla} \mathbf{v}
$$

where the first term on the RHS is the chemical force, the second term is the Coulomb force and the last is the viscous force.

The local expression of the second law requires the entropy source density to be locally positive definite (6). Extending Onsager's approach, in the neighbourhood of a local thermodynamic state where the scalar affinities $\mathcal{A}_{a}$ and the vectorial forces $\mathbf{F}_{\alpha}$ are sufficiently small, the entropy source density can be expressed formally as the sum of quadratic forms of $\mathcal{A}_{a}$ and $\mathbf{F}_{\alpha}$, which ensures that it is positive definite, i.e.

$$
\rho_{s}=\frac{1}{T}\left(\sum_{a, b} L_{a b} \mathcal{A}_{a} \mathcal{A}_{b}+\sum_{\alpha, \beta} L_{\alpha \beta} \cdot\left(\mathbf{F}_{\alpha} \odot \mathbf{F}_{\beta}\right)\right) \geqslant 0,
$$

where the phenomenological components are the Onsager matrix elements, which are of two different types: scalars $L_{\alpha \beta}$ and rank-2 tensors $L_{\alpha \beta}$. This is simply due to the fact that the entropy source density $\rho_{s}$ is a quadratic form of the generalised force density fields (136). The symmetries of the Onsager matrices are given by the Onsager reciprocity relations $[21,22]$, i.e.

$$
\begin{aligned}
L_{a b}\left(s, n_{A}, \mathbf{D}, \mathbf{B}\right) & =L_{a b}\left(s, n_{A}, \mathbf{D},-\mathbf{B}\right), \\
L_{\alpha \beta}\left(s, n_{A}, \mathbf{D}, \mathbf{B}\right) & =L_{\alpha \beta}\left(s, n_{A}, \mathbf{D},-\mathbf{B}\right),
\end{aligned}
$$

which cannot be derived within a thermodynamic approach but require a statistical treatment since they are a consequence of the time reversibility of the microscopic dynamics [14]. The inequality (136) has to hold for each part, in accordance with the Curie symmetry principle [23] that forbids couplings between fields of different nature, which implies that each quadratic form has to be positive definite.

Thus, the chemical reaction rate densities $\omega_{a}$ are related to the chemical affinities $\mathcal{A}_{b}$ through scalar linear phenomenological relations, i.e.

$$
\omega_{a}=\sum_{b} L_{a b} \mathcal{A}_{b}
$$

where the Onsager matrix has to satisfy,

$$
\frac{1}{T}\left\{L_{a b}\right\} \geqslant 0
$$

in order for the scalar quadratic form in the relation (136) to be positive definite. The scalar linear phenomenological relations (138) account for the dissipation due to the chemistry.
Similarly, the vectorial current densities $\mathbf{j}_{\alpha}$ are related to the vectorial forces $\mathbf{F}_{\beta}$ through vectorial linear phenomenological relations, i.e.

$$
\mathbf{j}_{\alpha}=\sum_{\beta} L_{\alpha \beta} \cdot \mathbf{F}_{\beta}
$$

where the Onsager matrix has to satisfy,

$$
\frac{1}{T}\left\{L_{\alpha \beta}\right\} \geqslant 0
$$

in order for the vectorial quadratic form in the relation (136) to be positive definite. The vectorial linear phenomenological relations (140) are expressed explicitly in terms of the currents densities $\mathbf{j}_{s}$ and $\mathbf{j}_{A}$ and forces densities $\mathbf{F}_{s}$ and $\mathbf{F}_{A}$ as,

$$
\left\{\begin{array}{l}
\mathbf{j}_{s}=L_{s s} \cdot(-\nabla T)+\sum_{B} L_{s B} \cdot \mathbf{F}_{B} \\
\mathbf{j}_{A}=L_{A s} \cdot(-\nabla T)+\sum_{B} L_{A B} \cdot \mathbf{F}_{B}
\end{array}\right.
$$

where the Onsager matrix (141) is positive definite, i.e.

$$
\frac{1}{T}\left(\begin{array}{ll}
L_{s s} & L_{s B} \\
L_{A s} & L_{A B}
\end{array}\right) \geqslant 0
$$

The vectorial linear phenomenological relations (142) account for the dissipation due to the transport.

We now discuss the physical meaning of the linear phenomenological relations. We analyse the linear phenomenological scalar and vectorial relations in turn. It is worth emphasising that although the scalar (138) and the vectorial (142) linear phenomenological relations are structurally independent, they are coupled through the intensive chemical fields $\mathcal{A}_{a}$ and $\mu_{A}$ as shown explicitly in relation (123).

\subsection{Scalar relations: chemistry}

\section{Chemical couplings}

We consider the thermodynamics of the local system $d \Sigma$ for a multicomponent fluid. The scalar linear relation (138) describes the chemical coupling [24] between the reactions $a$ and $b$, i.e.

$$
\omega_{a}\left(s, n_{A}, \mathbf{D}, \mathbf{B}\right)=\sum_{b} C_{a b}\left(s, n_{A}, \mathbf{D}, \mathbf{B}\right) \mathcal{A}_{b}\left(s, n_{A}, \mathbf{D}, \mathbf{B}\right),
$$

where $C_{a b}\left(s, n_{A}, \mathbf{D}, \mathbf{B}\right)=L_{a b}\left(s, n_{A}, \mathbf{D}, \mathbf{B}\right)$ are the chemical coupling coefficients, which are non-zero only if the reactions $a$ and $b$ occur in series or in parallel. 


\subsection{Vectorial relations: transport}

\subsubsection{Viscosity}

We consider the thermodynamics of the local system $d \Sigma$ for a single chemical substance $A$, consisting of electrically neutral elementary units, i.e. $q_{A}=0$, and that are homogeneously diluted, i.e. $\nabla \mu_{A}=\mathbf{0}$, in an electrically neutral and non-reactive viscous liquid solution at a uniform temperature, i.e. $\boldsymbol{\nabla} T=\mathbf{0}$. For a single chemical substance $\mathbf{v}=\mathbf{v}_{A}$. Thus, using relation (135), the linear phenomenological relation (142) reduces to,

$m_{A} \mathbf{v}_{A} \boldsymbol{\nabla} \mathbf{v}_{A}=-\boldsymbol{\eta}\left(s, n_{A}, \mathbf{D}, \mathbf{B}\right) \cdot G \mathbf{j}_{A}\left(s, n_{A}, \mathbf{D}, \mathbf{B}\right)$,

where

$$
\boldsymbol{\eta}\left(s, n_{A}, \mathbf{D}, \mathbf{B}\right) \equiv\left(G L_{A A}\right)^{-1},
$$

is the viscosity tensor of the liquid solution and $G$ is a geometric factor. The equation (145) is a tensorial generalisation of Stokes' law [25]. The symmetric viscosity tensor $\boldsymbol{\eta}$ can be split irreducibly into a bulk viscosity scalar $\eta$ and a shear viscosity trace-free tensor $\tilde{\boldsymbol{\eta}}$, i.e.

$$
\boldsymbol{\eta}=\eta \mathbb{1}+\tilde{\boldsymbol{\eta}}
$$

The bulk viscosity scalar $\eta$ describes viscous friction where the viscous force $m_{A} \mathbf{v}_{A} \boldsymbol{\nabla} \mathbf{v}_{A}$ is collinear to the matter flow $\mathbf{j}_{A}$. The shear viscosity trace-free tensor $\tilde{\boldsymbol{\eta}}$ describes viscous friction where the viscous force $m_{A} \mathbf{v}_{A} \boldsymbol{\nabla} \mathbf{v}_{A}$ is orthogonal to the matter flow $\mathbf{j}_{A}$.

In the rest of this section on transport, we consider only the case where there is no viscosity and no viscous force, i.e. $m_{A} \mathbf{v}_{A} \boldsymbol{\nabla} \mathbf{v}_{A}=\mathbf{0}$.

\subsubsection{Heat transport}

We consider the thermodynamics of the local system $d \Sigma$ consisting of chemical substances $A$ at rest in the local material frame of the fluid, i.e.

$$
\mathbf{j}_{A}\left(s, n_{A}, \mathbf{D}, \mathbf{B}\right)=\mathbf{0} \quad \forall A .
$$

In that case, the definition (132) of the heat current density $\mathbf{j}_{Q}\left(s, n_{A}, \mathbf{D}, \mathbf{B}\right)$ implies that the product of the temperature $T\left(s, n_{A}, \mathbf{D}, \mathbf{B}\right)$ and the vectorial linear relations (142) describes the heat transport, i.e.

$$
\mathbf{j}_{Q}\left(s, n_{A}, \mathbf{D}, \mathbf{B}\right)=-\boldsymbol{\kappa}\left(s, n_{A}, \mathbf{D}, \mathbf{B}\right) \cdot \nabla T\left(s, n_{A}, \mathbf{D}, \mathbf{B}\right),
$$

where $\boldsymbol{\kappa}\left(s, n_{A}, \mathbf{D}, \mathbf{B}\right)$ is the heat conductivity rank-2 tensor. The diagonal terms of the tensorial relation (148) represent Fourier's law [26], which can be inverted, i.e.

$\nabla T\left(s, n_{A}, \mathbf{D}, \mathbf{B}\right)=-\boldsymbol{\kappa}^{-1}\left(s, n_{A}, \mathbf{D}, \mathbf{B}\right) \cdot \mathbf{j}_{Q}\left(s, n_{A}, \mathbf{D}, \mathbf{B}\right)$.

The off-diagonal terms describe other phenomena. For instance, we consider a thin layer perpendicular to the $z$ axis with a heat current $\mathbf{j}_{Q}$ flowing along the $x$ axis. The tensorial relation (149) predicts the existence of a temperature gradient $\nabla T$ in the direction of the $y$ axis. In presence of a magnetic induction field $\mathbf{B}$ applied along the $z$ axis, this phenomenon is known as the Righi-Leduc effect $[27,28]$.

In the particular case where the temperature of the matter fields is uniform, i.e. $\boldsymbol{\nabla} T\left(s, n_{A}, \mathbf{0}, \mathbf{0}\right)=\mathbf{0}$, expression (117) for the temperature in terms of the electromagnetic fields, implies that Fourier's law (148) reduces to,

$$
\begin{aligned}
\mathbf{j}_{Q}\left(s, n_{A}, \mathbf{D}, \mathbf{B}\right)= & \boldsymbol{\kappa}\left(s, n_{A}, \mathbf{D}, \mathbf{B}\right) \\
& \times \nabla\left(\frac{\partial \varepsilon}{\partial s} \cdot(\mathbf{E} \odot \mathbf{E})+\frac{\partial \boldsymbol{\mu}}{\partial s} \cdot(\mathbf{H} \odot \mathbf{H})\right),
\end{aligned}
$$

which describes the heat flow of polarised and magnetised electrically neutral substances in the presence of nonuniform electromagnetic fields.

\subsubsection{Matter transport}

We consider the thermodynamics of the local system $d \Sigma$ consisting of electrically neutral chemical substances $B$ with a uniform temperature $T\left(s, n_{A}, \mathbf{D}, \mathbf{B}\right)$, i.e.

$$
q_{B}\left(n_{B}\right)=0 \quad \forall B \quad \text { and } \quad \nabla T\left(s, n_{A}, \mathbf{D}, \mathbf{B}\right)=\mathbf{0} .
$$

In that case, the vectorial linear relations (142) represent Fick's law [29] describing the chemical transport of the chemical substance $A$ with respect to the chemical substances $B$, i.e.

$$
\begin{aligned}
& \mathbf{j}_{A}\left(s, n_{A}, \mathbf{D}, \mathbf{B}\right)= \\
& \quad-\sum_{B} F_{A B}\left(s, n_{A}, \mathbf{D}, \mathbf{B}\right) \cdot \nabla \mu_{B}\left(s, n_{A}, \mathbf{D}, \mathbf{B}\right),
\end{aligned}
$$

where $F_{A B}\left(s, n_{A}, \mathbf{D}, \mathbf{B}\right)$ is the chemical transport rank-2 tensor.

In the particular case where the chemical potential of the matter fields is uniform, i.e. $\boldsymbol{\nabla} \mu_{A}\left(s, n_{A}, \mathbf{0}, \mathbf{0}\right)=\mathbf{0}$, expression (119) for the chemical potential in terms of the electromagnetic fields, implies that Fick's law (151) reduces to,

$$
\begin{aligned}
\mathbf{j}_{A}\left(s, n_{A}, \mathbf{D}, \mathbf{B}\right)= & \sum_{B} F_{A B}\left(s, n_{A}, \mathbf{D}, \mathbf{B}\right) \\
& \times \nabla\left(\frac{\partial \varepsilon}{\partial n_{B}} \cdot(\mathbf{E} \odot \mathbf{E})+\frac{\partial \boldsymbol{\mu}}{\partial n_{B}} \cdot(\mathbf{H} \odot \mathbf{H})\right),
\end{aligned}
$$

which describes optical tweezers [30], i.e. the flow of polarised and magnetised electrically neutral substances in the presence of non-uniform electromagnetic fields.

\subsubsection{Electric transport}

We consider the thermodynamics of the local system $d \Sigma$ with a uniform temperature $T\left(s, n_{A}, \mathbf{D}, \mathbf{B}\right)$ and uniform chemical potentials $\mu_{B}\left(s, n_{A}, \mathbf{D}, \mathbf{B}\right)$, i.e.

$$
\nabla T\left(s, n_{A}, \mathbf{D}, \mathbf{B}\right)=\mathbf{0} \quad \nabla \mu_{B}\left(s, n_{A}, \mathbf{D}, \mathbf{B}\right)=\mathbf{0} \quad \forall B
$$


In that case, the definition (105) of the diffusive electric current density $\mathbf{j}_{q}\left(s, n_{A}, \mathbf{D}, \mathbf{B}\right)$ implies that the product of the electric charge $q_{A}\left(n_{A}\right)$ per unit of chemical substance $A$ and the vectorial linear relations (142) describe the electric transport, i.e.

$$
\mathbf{j}_{q}\left(s, n_{A}, \mathbf{D}, \mathbf{B}\right)=\boldsymbol{\sigma}_{q}\left(s, n_{A}, \mathbf{D}, \mathbf{B}\right) \cdot \mathbf{E}\left(s, n_{A}, \mathbf{D}\right),
$$

where $\boldsymbol{\sigma}_{q}\left(s, n_{A}, \mathbf{D}, \mathbf{B}\right)$ is the electric conductivity rank-2 tensor. The diagonal terms of the tensorial relation (153) represent Ohm's law [31], which can be inverted, i.e.

$$
\mathbf{E}\left(s, n_{A}, \mathbf{D}\right)=\boldsymbol{\rho}_{q}\left(s, n_{A}, \mathbf{D}, \mathbf{B}\right) \cdot \mathbf{j}_{q}\left(s, n_{A}, \mathbf{D}, \mathbf{B}\right),
$$

where $\boldsymbol{\rho}_{q}\left(s, n_{A}, \mathbf{D}, \mathbf{B}\right)$ is the electric resistivity tensor defined as the inverse of the electric conductivity tensor, i.e.

$$
\boldsymbol{\rho}_{q}\left(s, n_{A}, \mathbf{D}, \mathbf{B}\right) \equiv \boldsymbol{\sigma}_{q}^{-1}\left(s, n_{A}, \mathbf{D}, \mathbf{B}\right) .
$$

The electric resistivity tensor depends on the magnetic induction field $\mathbf{B}$ and thus accounts for magnetoresistance [32].

The off-diagonal terms describe other phenomena. For instance, we consider a thin layer perpendicular to the $z$ axis with an electric current $\mathbf{j}_{q}$ flowing along the $x$ axis. The tensorial relation (149) predicts the existence of an electric field $\mathbf{E}$ in the direction of the $y$ axis. In presence of a magnetic induction field $\mathbf{B}$ applied along the $z$ axis, this phenomenon is known as the Hall effect [33].

\subsubsection{Thermochemical transport}

We consider the thermodynamics of the local system $d \Sigma$ consisting of electrically neutral chemical substances $A$ and $B$ at rest in the local material frame of the fluid, i.e.

$$
q_{A, B}\left(n_{A, B}\right)=0 \text { and } \mathbf{j}_{A, B}\left(s, n_{A, B}, \mathbf{D}, \mathbf{B}\right)=\mathbf{0} .
$$

In that case, the definition (132) of the heat current density $\mathbf{j}_{Q}\left(s, n_{A}, \mathbf{D}, \mathbf{B}\right)$ implies that the product of the temperature $T\left(s, n_{A}, \mathbf{D}, \mathbf{B}\right)$ and the vectorial linear relations (142) represent the Dufour effect [34] describing the thermochemical transport generated by a chemical gradient $\nabla \mu_{B}\left(s, n_{A}, \mathbf{D}, \mathbf{B}\right)$, i.e.

$$
\begin{aligned}
\mathbf{j}_{Q}\left(s, n_{A}, \mathbf{D}, \mathbf{B}\right)= & -\sum_{B} D_{B}\left(s, n_{A}, \mathbf{D}, \mathbf{B}\right) \\
& \times \nabla \mu_{B}\left(s, n_{A}, \mathbf{D}, \mathbf{B}\right),
\end{aligned}
$$

where $D_{B}\left(s, n_{A}, \mathbf{D}, \mathbf{B}\right)$ is the thermochemical transport rank-2 tensor of the electrically neutral chemical substance $B$. In the particular case where the chemical potential of the matter fields is uniform, i.e. $\nabla \mu_{A}\left(s, n_{A}, \mathbf{0}, \mathbf{0}\right)=\mathbf{0}$, the expression (119) for the chemical potential in terms of the electromagnetic fields, implies that the Dufour effect (155) reduces to,

$$
\begin{aligned}
\mathbf{j}_{Q}\left(s, n_{A}, \mathbf{D}, \mathbf{B}\right)= & \sum_{B} D_{B}\left(s, n_{A}, \mathbf{D}, \mathbf{B}\right) \\
& \times \nabla\left(\frac{\partial \varepsilon}{\partial n_{B}} \cdot(\mathbf{E} \odot \mathbf{E})+\frac{\partial \boldsymbol{\mu}}{\partial n_{B}} \cdot(\mathbf{H} \odot \mathbf{H})\right),
\end{aligned}
$$

which describes the heat flow of polarised and magnetised electrically neutral substances in the presence of nonuniform electromagnetic fields.

Moreover, when the system is at equilibrium, the vectorial linear relations (142) represent the Soret effect [35] describing the difference of chemical potential between the substances $A$ and $B$ generated by a temperature gradient $\nabla T\left(s, n_{A, B}, \mathbf{D}, \mathbf{B}\right)$, i.e.

$$
\begin{aligned}
\nabla(\Delta \mu)\left(s, n_{A, B}, \mathbf{D}, \mathbf{B}\right)= & -S\left(s, n_{A, B}, \mathbf{D}, \mathbf{B}\right) \\
& \times \nabla T\left(s, n_{A, B}, \mathbf{D}, \mathbf{B}\right),
\end{aligned}
$$

where $\Delta \mu\left(s, n_{A, B}, \mathbf{D}, \mathbf{B}\right)=\frac{\mu_{A}-\mu_{B}}{2}$ is the difference of chemical potential between the substances $A$ and $B$ and $S\left(s, n_{A, B}, \mathbf{D}, \mathbf{B}\right)$ is the Soret rank- 2 tensor. In the particular case where the temperature of the matter fields is uniform, i.e. $\boldsymbol{\nabla} T\left(s, n_{A}, \mathbf{0}, \mathbf{0}\right)=\mathbf{0}$, the expressions (117) and (119) respectively for the temperature and chemical potential in terms of the electromagnetic fields, imply that the Soret effect (157) reduces to,

$$
\begin{aligned}
\nabla(\Delta \mu)\left(s, n_{A, B}, \mathbf{D}, \mathbf{B}\right)= & S\left(s, n_{A, B}, \mathbf{D}, \mathbf{B}\right) \\
& \times \nabla\left(\frac{\partial \varepsilon}{\partial s} \cdot(\mathbf{E} \odot \mathbf{E})+\frac{\partial \boldsymbol{\mu}}{\partial s} \cdot(\mathbf{H} \odot \mathbf{H})\right),
\end{aligned}
$$

which describes the difference of chemical potential in the presence of non-uniform electromagnetic fields.

\subsubsection{Thermoelectric transport}

We consider the thermodynamics of the local system $d \Sigma$ with uniform chemical potentials $\mu_{B}\left(s, n_{A}, \mathbf{D}, \mathbf{B}\right)$ in the absence of chemical current densities $\mathbf{j}_{A}\left(s, n_{A}, \mathbf{D}, \mathbf{B}\right)$, i.e.

$$
\nabla \mu_{B}\left(s, n_{A}, \mathbf{D}, \mathbf{B}\right)=\mathbf{0} ; \quad \mathbf{j}_{A}\left(s, n_{A}, \mathbf{D}, \mathbf{B}\right)=\mathbf{0} \forall A, B .
$$

In that case, the definition (105) of the diffusive electric current density $\mathbf{j}_{q}\left(s, n_{A}, \mathbf{D}, \mathbf{B}\right)$ implies that the product of the electric charge $q_{A}\left(n_{A}\right)$ per unit of chemical substance $A$ and the vectorial linear relations (142) represent the Seebeck effect [36] describing the electric field generated by a temperature gradient $\nabla T$, i.e.

$$
\mathbf{E}\left(s, n_{A}, \mathbf{D}\right)=-\boldsymbol{\epsilon}\left(s, n_{A}, \mathbf{D}, \mathbf{B}\right) \cdot \nabla T\left(s, n_{A}, \mathbf{D}, \mathbf{B}\right),
$$

where $\boldsymbol{\epsilon}\left(s, n_{A}, \mathbf{D}, \mathbf{B}\right)$ is the Seebeck rank-2 tensor.

The off-diagonal terms describe other phenomena. For instance, we consider a thin layer perpendicular to the $z$ axis with a temperature gradient $\nabla T$ applied along the $x$ axis. The tensorial relation (159) predicts the existence of an electric field $\mathbf{E}$ in the direction of the $y$ axis. In presence of a magnetic induction field $\mathbf{B}$ applied along the $z$ axis, this phenomenon is known as the Nernst effect [37].

In the particular case where the temperature of the matter fields is uniform, i.e. $\boldsymbol{\nabla} T\left(s, n_{A}, \mathbf{0}, \mathbf{0}\right)=\mathbf{0}$, expression (117) for the temperature in terms of the electromagnetic fields, implies that the Seebeck effect (159) 
reduces to,

$$
\begin{aligned}
\mathbf{E}\left(s, n_{A}, \mathbf{D}\right)= & \boldsymbol{\epsilon}\left(s, n_{A}, \mathbf{D}, \mathbf{B}\right) \\
& \times \nabla\left(\frac{\partial \varepsilon}{\partial s} \cdot(\mathbf{E} \odot \mathbf{E})+\frac{\partial \boldsymbol{\mu}}{\partial s} \cdot(\mathbf{H} \odot \mathbf{H})\right),
\end{aligned}
$$

which describes the electric field induced in the presence of non-uniform electromagnetic fields.

\section{Conclusion}

We determined the local thermodynamics of a classical continuous medium consisting of electrically charged and reactive chemical substances in the presence of electromagnetic fields. In order to derive the local thermostatic equilibrium equation and the local reversible and irreversible thermodynamic evolution equations, we took into account the first and second laws of thermodynamics describing, respectively, the local evolution of the energy and the entropy, the chemical reactions describing the local evolution of the chemical substances, the second law of Newton in translation and in rotation describing, respectively, the local evolution of the momentum and the angular momentum, and Maxwell's equations describing the local evolution of the electromagnetic fields.

For a local thermodynamic equilibrium, we obtained explicit expressions for the temperature and the chemical potentials with additional terms arising from the presence of the electromagnetic fields and from the response of the matter to such fields. The analysis of the irreversible thermodynamics yields novel dissipative transport equations accounting in particular for the heat flow and the matter flows due to inhomogeneous electromagnetic fields and dielectric and magnetic matter responses.

The authors would like to honour the memory of E.C.G. Stückelberg von Breidenbach who, among other great achievements, developed a genuine dynamic theory of thermodynamics. They would also like to thank Klaus Maschke for insightful discussions.

\section{Appendix A: Thermodynamic axiomatic}

\section{A.1 First Law}

For every physical system $\Sigma$, there exists an extensive, scalar state function $E$, called energy such that if the system is isolated, the energy is conserved, i.e. $E$ is a constant observable. If the system is not isolated, then

$$
\frac{d E(t)}{d t}=P^{\mathrm{ext}}(t)
$$

where $P^{\text {ext }}(t)$ is the power corresponding to the energy exchanged per unit time with the exterior. The system is called "closed" if there is no exchange of matter, it is said "adiabatically closed" if additionally there is no heat exchange, and it is called "isolated" if there is no energy exchange at all, i.e. $P^{\text {ext }}(t)=0$.

\section{A.2 Second Law}

For every system $\Sigma$, there exists an extensive, scalar state variable $S$, called entropy, which obeys the following two conditions [5]:

- evolution condition:

if the system is adiabatically closed, the entropy $S(t)$ is a monotonous increasing function of time, i.e.

$$
\frac{d S(t)}{d t}=\Pi_{s}(t) \geqslant 0
$$

where $\Pi_{s}(t)$ is the entropy production rate accounting for the irreversibility of the system;

- equilibrium condition:

if the system is isolated, the entropy tends towards a finite local maximum, compatible with the constraints as time tends to infinity.

\section{A.3 Continuity equation for an extensive state function}

A thermodynamic system $\Sigma$ is defined by a set of physical properties. The time evolution of the system is described by a set of time-dependent states variables accounting for these properties. The second law of thermodynamics requires the existence of a state variable entropy $S(t)$ for every system. Thus, the state of a system is defined by the state variable entropy $S(t)$ and a finite set of $n$ time-dependent state variables denoted $X_{1}(t)$, $X_{2}(t), \ldots, X_{n}(t)$. The state of the global thermodynamic system $\Sigma$, compatible with the constraints, is defined formally by the following set of state variables, i.e. $\left\{S(t), X_{1}(t), X_{2}(t), \ldots, X_{n}(t)\right\}$.

The physical properties $F(t)$ characterising the system dynamics are called "state functions", since they are functions of state variables and time $t$, i.e.

$$
F(t) \equiv F\left(S(t), X_{1}(t), X_{2}(t), \ldots, X_{n}(t), t\right) .
$$

Note that the state variables can be treated as trivial state functions $F(t)$ that are independent of the other state variables.

A thermodynamic system of finite volume $V$ is characterised by a large number of elementary constituents, distributed within a none-empty enclosure $\partial \Sigma$ of surface $\partial V$, which is a smooth manifold. Although a macroscopic medium is a discrete system consisting of a finite number of particles, it can be idealised as a continuous system on a sufficiently large scale. On a scale between the microscopic and macroscopic scales, the system is locally sufficiently large from a microscopic perspective to be insensitive to quantum fluctuations and thus to be treated classically, but it is sufficiently small from a macroscopic perspective to be considered as infinitesimal. The neighbourhood of every point of the system belongs to a local thermodynamic system $d \Sigma$, which is considered as homogeneous and continuous on a microscopic scale and pointlike on a macroscopic scale. 
The dynamics of a local system $d \Sigma$ can be described either in a fixed spatial frame, which is a Eulerian frame, or in a moving local material frame, which is a Lagrangian frame. The Eulerian frame shall be referred to simply as the spatial frame and the local Lagrangian frame as the local material frame. The space and time coordinates, which parametrise a physical event, are $\left(\mathbf{x}^{\prime}, t^{\prime}\right)$ in the spatial frame and $(\mathbf{x}, t)$ in the local material frame. The spatial points $\mathbf{x}$ are fixed with respect to the spatial frame, whereas the material points $\mathbf{x}^{\prime}$ are moving with the matter. In a non-relativistic framework, time is an invariant parameter, i.e. $t=t^{\prime}$. However, it is necessary to distinguish formally the time parameter in the two frames, since the partial time derivatives are not equal, i.e. $\partial_{t} \neq \partial_{t^{\prime}}$, as shown in Appendix A.4.

In order to determine the dynamics in the system $\Sigma$, we start by describing the matter in the spatial frame. In the spatial frame, the thermodynamic state of the local thermodynamic system $d \Sigma$ is characterised by the entropy density field $s^{\prime}\left(\mathbf{x}^{\prime}, t^{\prime}\right)$ and a set of $n$ fields $x_{1}^{\prime}\left(\mathbf{x}^{\prime}, t^{\prime}\right), x_{2}^{\prime}\left(\mathbf{x}^{\prime}, t^{\prime}\right), \ldots, x_{n}^{\prime}\left(\mathbf{x}^{\prime}, t^{\prime}\right)$. The local thermodynamic state compatible with the constraints is defined formally by the following set of state fields, i.e. $\left\{s^{\prime}\left(\mathbf{x}^{\prime}, t^{\prime}\right), x_{1}^{\prime}\left(\mathbf{x}^{\prime}, t^{\prime}\right), \ldots, x_{n}^{\prime}\left(\mathbf{x}^{\prime}, t^{\prime}\right)\right\}$.

The physical properties $f^{\prime}\left(\mathbf{x}^{\prime}, t^{\prime}\right)$ characterising the local dynamics of the system are called "state function densities". These properties are functions of the state fields, position $\mathbf{x}^{\prime}$ and time $t^{\prime}$, i.e.

$$
f^{\prime}\left(\mathbf{x}^{\prime}, t^{\prime}\right) \equiv f^{\prime}\left(s^{\prime}\left(\mathbf{x}^{\prime}, t^{\prime}\right), x_{1}^{\prime}\left(\mathbf{x}^{\prime}, t^{\prime}\right), \ldots, x_{n}^{\prime}\left(\mathbf{x}^{\prime}, t^{\prime}\right), \mathbf{x}^{\prime}, t^{\prime}\right)
$$

Note that the state fields can be treated as trivial state functions densities $f^{\prime}\left(\mathbf{x}^{\prime}, t^{\prime}\right)$ that are independent of the other state fields.

An arbitrary extensive scalar state function $F\left(t^{\prime}\right)$ is related to the corresponding state function density $f^{\prime}\left(\mathbf{x}^{\prime}, t^{\prime}\right)$ by an integral over the volume, i.e.

$$
F\left(t^{\prime}\right)=\int_{V} d V\left(\mathbf{x}^{\prime}, t^{\prime}\right) f^{\prime}\left(\mathbf{x}^{\prime}, t^{\prime}\right) .
$$

In order to obtain the continuity equation for the extensive scalar state function $F\left(t^{\prime}\right)$, we vary $F\left(t^{\prime}\right)$. The infinitesimal element $\delta F\left(t^{\prime}\right)$ is the sum of a bulk term and a boundary term, i.e.

$$
\delta F\left(t^{\prime}\right)=\int_{V} d V\left(\mathbf{x}^{\prime}, t^{\prime}\right) \delta f^{\prime}\left(\mathbf{x}^{\prime}, t^{\prime}\right)+\int_{\partial V} \delta d V\left(\mathbf{x}^{\prime}, t^{\prime}\right) f^{\prime}\left(\mathbf{x}^{\prime}, t^{\prime}\right),
$$

where the first integrand represents the variation of the local state function density field $\delta f^{\prime}\left(\mathbf{x}^{\prime}, t^{\prime}\right)$ and the second integrand represents the variation of the local infinitesimal volume $\delta d V\left(\mathbf{x}^{\prime}, t^{\prime}\right)$. The latter is the inner product of the contravariant displacement vector field $\delta \mathbf{r}\left(\mathbf{x}^{\prime}, t^{\prime}\right)$ and the infinitesimal covariant surface element vector field $d \boldsymbol{\sigma}\left(\mathbf{x}^{\prime}, t^{\prime}\right)$ according to,

$$
\delta d V\left(\mathbf{x}^{\prime}, t^{\prime}\right)=d \boldsymbol{\sigma}\left(\mathbf{x}^{\prime}, t^{\prime}\right) \delta \mathbf{r}\left(\mathbf{x}^{\prime}, t^{\prime}\right) .
$$

The time derivative of the extensive scalar state functional $d_{t^{\prime}} F\left(t^{\prime}\right)$, the partial time derivative of the state function density field $\partial_{t} f\left(\mathbf{x}^{\prime}, t^{\prime}\right)$ and the matter velocity field $\mathbf{v}\left(\mathbf{x}^{\prime}, t^{\prime}\right)$ are respectively defined as,

$$
\begin{aligned}
d_{t^{\prime}} F\left(t^{\prime}\right) & \equiv \frac{d F\left(t^{\prime}\right)}{d t}=\lim _{\delta t^{\prime} \rightarrow 0} \frac{\delta F\left(t^{\prime}\right)}{\delta t^{\prime}} \\
\partial_{t^{\prime}} f^{\prime}\left(\mathbf{x}^{\prime}, t^{\prime}\right) & \equiv \frac{\partial f^{\prime}\left(\mathbf{x}^{\prime}, t^{\prime}\right)}{\partial t^{\prime}}=\lim _{\delta t^{\prime} \rightarrow 0} \frac{\delta f^{\prime}\left(\mathbf{x}^{\prime}, t^{\prime}\right)}{\delta t^{\prime}} \\
\mathbf{v}\left(\mathbf{x}^{\prime}, t^{\prime}\right) & \equiv \lim _{\delta t^{\prime} \rightarrow 0} \frac{\delta \mathbf{r}\left(\mathbf{x}^{\prime}, t^{\prime}\right)}{\delta t} .
\end{aligned}
$$

Thus, the time derivative of the extensive scalar state function $d_{t^{\prime}} F\left(t^{\prime}\right)$ is found to be,

$$
\begin{aligned}
d_{t^{\prime}} F\left(t^{\prime}\right)= & \int_{V} d V\left(\mathbf{x}^{\prime}, t^{\prime}\right) \partial_{t^{\prime}} f^{\prime}\left(\mathbf{x}^{\prime}, t^{\prime}\right) \\
& +\int_{\partial V} d \boldsymbol{\sigma}\left(\mathbf{x}^{\prime}, t^{\prime}\right) \cdot \mathbf{v}\left(\mathbf{x}^{\prime}, t^{\prime}\right) f^{\prime}\left(\mathbf{x}^{\prime}, t^{\prime}\right) .
\end{aligned}
$$

Using Gauss' theorem, this time derivative (A.9) can be recast as,

$d_{t^{\prime}} F\left(t^{\prime}\right)=\int_{V} d V\left(\mathbf{x}^{\prime}, t^{\prime}\right)\left[\partial_{t^{\prime}} f^{\prime}\left(\mathbf{x}^{\prime}, t^{\prime}\right)+\nabla^{\prime} \cdot\left(f^{\prime}\left(\mathbf{x}^{\prime}, t^{\prime}\right) \mathbf{v}\left(\mathbf{x}^{\prime}, t^{\prime}\right)\right)\right]$.

The integral expression (A.10) for the time derivative of the state function was derived purely on a mathematical level. We now describe the two physical causes of the rate of change of the scalar state function $F\left(t^{\prime}\right)$ that accounts for a physical property of the system. The first is due to the flux of $F\left(t^{\prime}\right)$ into or out of the system. This flux is generated by the contravariant current density vector field $\mathbf{j}_{f^{\prime}}^{\prime}\left(\mathbf{x}^{\prime}, t^{\prime}\right)$. The second is due to the production of $F\left(t^{\prime}\right)$ within the system. This production is generated by the source density field $\rho_{f^{\prime}}^{\prime}\left(\mathbf{x}^{\prime}, t^{\prime}\right)$. By convention, the current density field $\mathbf{j}_{f^{\prime}}^{\prime}\left(\mathbf{x}^{\prime}, t^{\prime}\right)$ is positive definite for an influx and the covariant infinitesimal surface element $d \boldsymbol{\sigma}$ is pointing out. Thus, on a physical level, the time derivative of the scalar state function $F\left(t^{\prime}\right)$ is given by,

$$
d_{t^{\prime}} F\left(t^{\prime}\right)=\int_{V} d V\left(\mathbf{x}^{\prime}, t^{\prime}\right) \rho_{f^{\prime}}^{\prime}(\mathbf{x}, t)-\int_{\partial V} d \boldsymbol{\sigma}\left(\mathbf{x}^{\prime}, t^{\prime}\right) \cdot \mathbf{j}_{f^{\prime}}^{\prime}\left(\mathbf{x}^{\prime}, t^{\prime}\right),
$$

where the negative sign in front of the second integral is due to the fact that the covariant vector $d \boldsymbol{\sigma}\left(\mathbf{x}^{\prime}, t^{\prime}\right)$ and the contravariant vector $\mathbf{j}_{f^{\prime}}^{\prime}\left(\mathbf{x}^{\prime}, t^{\prime}\right)$ point in opposite directions. Using Gauss' theorem, this time derivative (A.11) can be recast as,

$$
d_{t^{\prime}} F\left(t^{\prime}\right)=\int_{V} d V\left(\mathbf{x}^{\prime}, t^{\prime}\right)\left[\rho_{f^{\prime}}^{\prime}\left(\mathbf{x}^{\prime}, t^{\prime}\right)-\nabla^{\prime} \cdot \mathbf{j}_{f^{\prime}}^{\prime}\left(\mathbf{x}^{\prime}, t^{\prime}\right)\right]
$$

By identifying the mathematical (A.10) and physical (A.12) expressions for the time derivative of the state function $F\left(t^{\prime}\right)$, the integrands satisfy locally the continuity equation for $F\left(t^{\prime}\right)$ in the spatial frame given by,

$$
\partial_{t^{\prime}} f^{\prime}\left(\mathbf{x}^{\prime}, t^{\prime}\right)+\nabla^{\prime}\left(f^{\prime}\left(\mathbf{x}^{\prime}, t^{\prime}\right) \mathbf{v}\left(\mathbf{x}^{\prime}, t^{\prime}\right)+\mathbf{j}_{f^{\prime}}^{\prime}\left(\mathbf{x}^{\prime}, t^{\prime}\right)\right)=\rho_{f^{\prime}}^{\prime}\left(\mathbf{x}^{\prime}, t^{\prime}\right)
$$

where $f^{\prime}\left(\mathbf{x}^{\prime}, t^{\prime}\right) \mathbf{v}\left(\mathbf{x}^{\prime}, t^{\prime}\right)$ is the convective current density field and $\mathbf{j}_{f^{\prime}}^{\prime}\left(\mathbf{x}^{\prime}, t^{\prime}\right)$ is the diffusive current density field. 
The convective current density field is defined as the current density due to the motion of the matter with respect to the spatial frame. The diffusive current density field is defined as the current density in the local material frame.

At this point, we seek to express the continuity equation (A.13) in the local material frame. The extensive physical property $F(t)$ of global system is related to the corresponding physical property $f(\mathbf{x}, t)$ of the local system in the material frame by

$$
F(t)=\int_{V} d V(\mathbf{x}, t) f(\mathbf{x}, t) .
$$

The time derivative $d_{t} F(t)$ of the extensive physical property of the global system in the material frame is related to the source density field $\rho_{f}(\mathbf{x}, t)$ and the current density field $\mathbf{j}_{f}(\mathbf{x}, t)$ by,

$$
d_{t} F(t)=\int_{V} d V(\mathbf{x}, t)\left[\rho_{f}(\mathbf{x}, t)-\nabla \cdot \mathbf{j}_{f}(\mathbf{x}, t)\right] .
$$

In a non-relativistic framework, the time $t^{\prime}$ in the spatial frame coincides with the time $t$ in the local material frame, i.e. $t=t^{\prime}$, which implies that,

$$
\begin{aligned}
& F(t)=F\left(t^{\prime}\right), \\
& d_{t} F(t)=d_{t^{\prime}} F\left(t^{\prime}\right),
\end{aligned}
$$

and by comparison between the expressions (A.5) and (A.14) and the expressions (A.12) and (A.15), respectively, it implies in turn that,

$$
\begin{aligned}
f(\mathbf{x}, t) & =f^{\prime}\left(\mathbf{x}^{\prime}, t^{\prime}\right), \\
\rho_{f}(\mathbf{x}, t) & =\rho_{f^{\prime}}^{\prime}\left(\mathbf{x}^{\prime}, t^{\prime}\right), \\
\mathbf{j}_{f}(\mathbf{x}, t) & =\mathbf{j}_{f^{\prime}}^{\prime}\left(\mathbf{x}^{\prime}, t^{\prime}\right) .
\end{aligned}
$$

The time derivative in the local material frame is defined as,

$$
\dot{f}(\mathbf{x}, t) \equiv \partial_{t} f(\mathbf{x}, t) .
$$

As shown in Appendix A.4, for every physical event parametrised by the coordinates $\left(\mathbf{x}^{\prime}, t^{\prime}\right)$ in the spatial frame, the spatial and temporal differential operators in the local material frame are related to the corresponding operators in the spatial frame through a Galilean transformation of velocity $\mathbf{v}\left(\mathbf{x}^{\prime}, t^{\prime}\right)$ associated to the event, i.e.

$$
\begin{aligned}
\nabla & =\nabla^{\prime}, \\
\partial_{t} & =\partial_{t^{\prime}}+\mathbf{v}\left(\mathbf{x}^{\prime}, t^{\prime}\right) \cdot \nabla^{\prime} .
\end{aligned}
$$

The first relation (A.17) and the transformation law (A.19) for the partial time differential operator under the action of a Galilean transformation between the spatial frame and the local material frame imply that,

$$
\dot{f}(\mathbf{x}, t) \equiv \partial_{t^{\prime}} f^{\prime}\left(\mathbf{x}^{\prime}, t^{\prime}\right)+\mathbf{v}\left(\mathbf{x}^{\prime}, t^{\prime}\right) \cdot \nabla^{\prime} f^{\prime}\left(\mathbf{x}^{\prime}, t^{\prime}\right) .
$$

Using the relations (A.17), (A.19) and (A.20), the vectorial identities,

$$
\begin{aligned}
\nabla^{\prime} \cdot\left(f^{\prime}\left(\mathbf{x}^{\prime}, t^{\prime}\right) \mathbf{v}\left(\mathbf{x}^{\prime}, t^{\prime}\right)\right)= & \mathbf{v}\left(\mathbf{x}^{\prime}, t^{\prime}\right) \cdot \nabla^{\prime} f^{\prime}\left(\mathbf{x}^{\prime}, t^{\prime}\right) \\
& +\left(\nabla^{\prime} \cdot \mathbf{v}\left(\mathbf{x}^{\prime}, t^{\prime}\right)\right) f^{\prime}\left(\mathbf{x}^{\prime}, t^{\prime}\right)
\end{aligned}
$$

and $\mathbf{v}\left(\mathbf{x}^{\prime}, t^{\prime}\right)=\mathbf{v}\left(\mathbf{x}^{\prime}, t\right)$, the continuity equation (A.13) for $F(t)$ expressed in the spatial frame is recast in the local material frame according to,

$$
\dot{f}(\mathbf{x}, t)+\left(\boldsymbol{\nabla} \cdot \mathbf{v}\left(\mathbf{x}^{\prime}, t\right)\right) f(\mathbf{x}, t)+\nabla \cdot \mathbf{j}_{f}(\mathbf{x}, t)=\rho_{f}(\mathbf{x}, t),
$$

where the velocity field $\mathbf{v}\left(\mathbf{x}^{\prime}, t\right)$ is expressed in terms of the spatial coordinate $\mathbf{x}^{\prime}$ of the spatial frame since it represents the relative velocity of the local material frame with respect to the spatial frame. Thus, the velocity field vanishes in the local material field, i.e. $\mathbf{v}(\mathbf{x}, t)=\mathbf{0}$.

In our analysis, we derived the continuity equation (A.22) for a scalar state function $F(t)$. The generalisation to a tensor state function of arbitrary rank is straightforward. However, we shall limit our discussion to a vectorial state function $\mathbf{F}(t)$. The corresponding continuity equation in the local material frame is given by,

$\dot{\mathbf{f}}(\mathbf{x}, t)+\left(\boldsymbol{\nabla} \cdot \mathbf{v}\left(\mathbf{x}^{\prime}, t\right)\right) \mathbf{f}(\mathbf{x}, t)+\nabla \cdot j_{\mathbf{f}}(\mathbf{x}, t)=\rho_{\mathbf{f}}(\mathbf{x}, t)$,

where $\mathbf{f}(\mathbf{x}, t)$ is the state function density vector field, $j_{\mathbf{f}}(\mathbf{x}, t)$ is the diffusive current density tensor field and $\boldsymbol{\rho}_{\mathbf{f}}(\mathbf{x}, t)$ is the source density vector field. Note that the vectorial divergence of the diffusive current density tensor field $\boldsymbol{\nabla} \cdot j_{\mathbf{f}}(\mathbf{x}, t)$ is written explicitly in components as,

$$
\boldsymbol{\nabla} \cdot j_{\mathbf{f}}(\mathbf{x}, t)^{i}=\partial_{j} j_{\mathbf{f}}^{j i}(\mathbf{x}, t),
$$

using the Einstein implicit summation convention.

\section{A.4 Galilean transformation relations}

The coordinate systems of two inertial frames with a non-relativistic relative velocity are related by a Galilean transformation. The matter is non-relativistic and the spatial frame is an inertial frame with space-time coordinates $\left(\mathbf{x}^{\prime}, t^{\prime}\right)$. For every physical event $(\mathbf{x}, t)$ in the local material frame, there exists an inertial frame with space-time coordinates $(\mathbf{x}, t)$ that coincides with the local material frame. The space-time coordinates of the two inertial frames are related by the Galilean transformation,

$$
\begin{aligned}
\mathbf{x} & =\mathbf{x}^{\prime}-\mathbf{v} t^{\prime}, \\
t & =t^{\prime},
\end{aligned}
$$

where $\mathbf{v}$ is the non-relativistic relative velocity between the coinciding inertial frame and the spatial frame. Although we restrict our dynamical analysis to the nonrelativistic Galilean group, it is useful to introduce the four-vector notation in order to determine the frame transformation relations for vectors and tensors. In particular, the four-vector position in the spatial frame is denoted $x^{\prime}=\left({x^{\prime}}^{0}, \mathbf{x}^{\prime}\right)$ where the first coordinate, $x^{\prime 0}=c t^{\prime}$, is the product of the time coordinate $t^{\prime}$ with the dynamical constant $c$ representing the speed of light in the vacuum. In the spatial frame, an arbitrary contravariant four-vector is denoted $A^{\prime}=\left(A^{\prime 0}, \mathbf{A}^{\prime}\right)$ and an arbitrary covariant fourvector $B^{\prime}=\left(B_{0}^{\prime}, \mathbf{B}^{\prime}\right)$. Under a general coordinate transformation, the components of a contravariant four-vector $A^{\prime \mu}$ 
and a covariant four-vector $B_{\mu}^{\prime}$ transform respectively according to [38],

$$
\begin{aligned}
& A^{\mu}=\frac{\partial x^{\mu}}{\partial x^{\prime \nu}} A^{\prime \nu}, \\
& B_{\mu}=\frac{\partial x^{\prime \nu}}{\partial x^{\mu}} B_{\nu}^{\prime},
\end{aligned}
$$

where we used the summation convention of Einstein for the indices $\mu, \nu=\{0, \ldots, 3\}$. Thus, in the particular case of a Galilean transformation (A.24), a contravariant vector $\mathbf{A}^{\prime}$ and the associated scalar $A^{\prime 0}$ transform as,

$$
\begin{aligned}
\mathbf{A} & =\mathbf{A}^{\prime}-\frac{1}{c} A^{\prime 0} \mathbf{v}, \\
A^{0} & =A^{\prime 0}
\end{aligned}
$$

a covariant vector $\mathbf{B}^{\prime}$ and the associated scalar $B_{0}^{\prime}$ transform as,

$$
\begin{aligned}
\mathbf{B} & =\mathbf{B}^{\prime}, \\
B_{0} & =B_{0}^{\prime}+\frac{1}{c} \mathbf{v} \cdot \mathbf{B}^{\prime} .
\end{aligned}
$$

Note that the terms $A^{\prime 0} \mathbf{v} / c$ and $\mathbf{v} \cdot \mathbf{B}^{\prime} / c$ are not necessarily negligible in the Galilean limit, i.e. $|\mathbf{v}| / c \rightarrow 0$, since $A^{\prime 0}$ and $\mathbf{B}^{\prime}$ can be proportional to $c$.

The gradient $\nabla^{\prime}$ is a covariant vector, and the time component of the four-gradient covariant vector is the differential operator $c \partial_{t^{\prime}}$. From the Galilean transformation relation for a covariant vector (A.26), we deduce the Galilean transformation relation for this covariant vector according to,

$$
\begin{aligned}
& \boldsymbol{\nabla}=\nabla^{\prime} \\
& \partial_{t}=\partial_{t^{\prime}}+\mathbf{v} \cdot \nabla^{\prime},
\end{aligned}
$$

which explicitly shows that the gradient $\nabla^{\prime}$ is frameindependent and the time derivative is frame-dependent. From the first transformation in (A.27), it follows that the divergence and curl operators are also frame-independent.

It is worth mentioning that since Galilean frame transformations preserve lengths, volumes are Galilean invariants. Thus, any contravariant vector density a or any covariant vector density $\mathbf{b}$ transforms under a Galilean transformation respectively like the corresponding contravariant vector $\mathbf{A}$ or covariant vector $\mathbf{B}$.

The electric charge is a Galilean invariant, the electric charge density field $q$ is a Galilean frame-independent field, i.e. $q=q^{\prime}$. The electric current density $\mathbf{j}_{q}$ is a contravariant vector, which transforms under a Galilean transformation of velocity $\mathbf{v}$ as (A.25),

$$
\mathbf{j}_{q}=\mathbf{j}_{q}^{\prime}-q^{\prime} \mathbf{v}
$$

where the time component of the electric current density contravariant four-vector is the charge density field, $j_{q}^{\prime 0}=c q^{\prime}$.

In order to determine the Galilean frame transformations for the electromagnetic fields, we recast the electromagnetic fields $\mathbf{D}^{\prime}, \mathbf{B}^{\prime}, \mathbf{E}^{\prime}$ and $\mathbf{H}^{\prime}$ in the spatial frame into two antisymmetric tensors of rank-2. The first tensor is the covariant electromagnetic tensor $F^{\prime}$. The non-diagonal components of this antisymmetric tensor, denoted $F_{\mu \nu}^{\prime}$, are related to the components of the covariant electric field $E_{i}^{\prime}$ and to the components of the contravariant magnetic induction field, $B^{\prime k}$ respectively by [39],

$$
\begin{aligned}
F^{\prime}{ }_{i 0} & =\frac{1}{c} E_{i}^{\prime}, \\
F^{\prime}{ }_{i j} & =\varepsilon_{i j k} B^{\prime k},
\end{aligned}
$$

where $\mu=\{0, \ldots, 3\}, i=\{1,2,3\}$. The second tensor is the contravariant dielectromagnetic tensor $D^{\prime}$. Similarly, the non-diagonal components of this antisymmetric tensor, denoted $D^{\prime \mu \nu}$, are related to the components of the contravariant electric displacement field $D^{\prime i}$ and to the components of the covariant magnetic field $H_{k}^{\prime}$, respectively by [39],

$$
\begin{aligned}
& {D^{\prime 0 i}}^{0 i}=c D^{\prime i}, \\
& {D^{\prime i j}}^{i j}=\varepsilon^{i j k} H_{k}^{\prime} .
\end{aligned}
$$

Under a general coordinate transformation, the covariant components of the electromagnetic tensor $F_{\mu \nu}$ and the contravariant components of the dielectromagnetic tensor $D^{\mu \nu}$ transform respectively as [38],

$$
\begin{aligned}
F_{\mu \nu} & =\frac{\partial x^{\prime \rho}}{\partial x^{\mu}} \frac{\partial x^{\prime \sigma}}{\partial x^{\nu}} F_{\rho \sigma}^{\prime}, \\
D^{\mu \nu} & =\frac{\partial x^{\mu}}{\partial x^{\prime \rho}} \frac{\partial x^{\nu}}{\partial x^{\prime \sigma}} D^{\prime \rho \sigma} .
\end{aligned}
$$

Thus, in the particular case of a Galilean transformation (A.24) of constant velocity $\mathbf{v}$, the fields $\mathbf{D}^{\prime}, \mathbf{E}^{\prime}, \mathbf{B}^{\prime}$, $\mathbf{H}^{\prime}$, transform as,

$$
\begin{aligned}
\mathbf{D} & =\mathbf{D}^{\prime}, \\
\mathbf{B} & =\mathbf{B}^{\prime}, \\
\mathbf{E} & =\mathbf{E}^{\prime}+\mathbf{v} \times \mathbf{B}^{\prime}, \\
\mathbf{H} & =\mathbf{H}^{\prime}-\mathbf{v} \times \mathbf{D}^{\prime} .
\end{aligned}
$$

The Galilean transformations (A.32) show explicitly that the fields $\mathbf{D}$ and $\mathbf{B}$ are frame-independent fields whereas the fields $\mathbf{E}$ and $\mathbf{H}$ are frame-dependent. It is worth mentioning that these Galilean transformation laws differ from the ones obtained by de Groot and Mazur [40]. It is straightforward to show that their relations lead to a contradiction since they imply that the Lorentz force is not Galilean frame-independent, which is physically unacceptable in a non-relativistic framework.

\section{References}

1. S. Carnot, Réflexions sur la puissance motrice du feu et sur les machines propres à développer cette puissance (Bachelier, Paris, 1824)

2. C. Ferrari, C. Gruber, Eur. J. Phys. 31, 1159 (2010) 
3. C. Eckart, Phys. Rev. 58, 267 (1940)

4. C. Eckart, Phys. Rev. 58, 269 (1940)

5. E.C.G. Stückelberg von Breidenbach, P.B. Scheurer, Thermocinétique Phénoménologique Galiléenne (Birkhauser, Basel, 1974)

6. I. Prigogine, Introduction to the thermodynamics of irreversible processes (Interscience, New York, 1961)

7. I. Müller, Thermodynamics (Pitman, Boston, 1985)

8. W. Muschik, L. Restuccia, Arch. Appl. Mech. 78, 837 (2008)

9. W. Muschik, C. Papenfuss, H. Ehrentraut, J. NonNewtonian Fluid Mech. 96, 255 (2001)

10. W. Muschik, J. Theor. Appl. Mech. 50, 807 (2012)

11. W. Muschik, J. Non Equilib. Thermodyn. 33, 165 (2008)

12. W. Muschik, L. Restuccia, Tech. Mech. 22, 152 (2002)

13. M. Le Bellac, J. Lévy-Leblond, Nuovo Cim. B 14, 217 (1973)

14. L. Onsager, Phys. Rev. 37, 405 (1931)

15. L. Onsager, Phys. Rev. 38, 2265 (1931)

16. D. van Dantzig, Proc. Cam. Phil. Soc. 30, 421 (1934)

17. C.W. Misner, K.S. Thorne, J.A. Wheeler, Gravitation (W. H. Freeman and company, New York, 1973)

18. F.W. Hehl, Y.N. Obukhov, arXiv:physics/0005084v2 [physics.class-ph] (2000)

19. L.D. Landau, E.M. Lifshits, The electrodynamics of continuous media, 2nd edn. (Elsevier, Amsterdam, 1982)

20. J.A. Stratton, Electromagnetic theory, 1st edn. (John Wiley and Sons, New York, 1941)

21. P. Mazur, S. De Groot, Physica 19, 961 (1953)
22. H.B. Callen, Thermodynamics and an Introduction to Thermostatistics, 2nd edn. (John Wiley and Sons, New York, 1985)

23. P. Curie, J. Phys. Theor. Appl. 3, 393 (1894)

24. C.J. Pings, E.B. Nebeker, Ind. Eng. Chem. Fund. 4, 376 (1965)

25. G.G. Stokes, Cambridge Philos. Trans. 9, 8 (1851)

26. J. Fourier, Théorie analytique de la chaleur (Firmin Didot, Paris, 1822)

27. A. Righi, Mem. Acc. Lincei 4, 433 (1887)

28. M.A. Leduc, J. Phys. 6, 378 (1887)

29. A. Fick, Pog. Ann. Phys. 94, 59 (1855)

30. A. Ashkin, Phys. Rev. Lett. 24, 156 (1970)

31. G.S. Ohm, Die glavanische Kette (T.H. Riemann, Berlin, 1827)

32. W. Thomson, Proc. R. Soc. London 8, 546 (1894)

33. E.H. Hall, Am. J. Math. 2, 287 (1879)

34. L. Dufour, Ann. Phys. 28, 490 (1873)

35. C. Soret, Archives des Sciences Physiques et Naturelles 2, 48 (1879)

36. T.J. Seebeck, Abh. Akad. Wiss. Berlin 289-346 (1822)

37. W. Nernst, Wien. Ber. 94, 560 (1886)

38. S. Weinberg, Gravitation and Cosmology: Principles and Applications of the General Theory of Relativity (John Wiley and Sons, New York, 1972)

39. F. Reuse, Electrodynamique et optique quantiques (Presses Polytechniques Universitaires Romandes, Lausanne, 2007)

40. S.R. de Groot, P. Mazur, Non-equilibrium thermodynamics (Dover, New York, 1984) 